\title{
1 HSF2 protects against proteotoxicity by maintaining cell-cell adhesion
}

3 Jenny Joutsen ${ }^{1,2^{*}}$, Alejandro J. Da Silva ${ }^{1,2^{*}}$, Marek A. Budzynski ${ }^{1,2}$, Jens C. Luoto ${ }^{1,2}$, Aurelie de

4 Thonel $^{3,4,5}$, Jean-Paul Concordet ${ }^{6}$, Valérie Mezger $^{3,4,5}$, Délara Sabéran-Djoneidi ${ }^{3,4,5}$, Eva

5 Henriksson ${ }^{1,2}$, Lea Sistonen ${ }^{1,2, \#}$

$7{ }^{1}$ Faculty of Science and Engineering, Cell Biology, Åbo Akademi University, Tykistökatu 6,

820520 Turku, Finland

92 Turku Centre for Biotechnology, University of Turku and Åbo Akademi University, Tykistökatu

10 6, 20520 Turku, Finland

$11{ }^{3}$ CNRS, UMR 7216 "Epigenetic and Cell Fate", 75250 Paris Cedex 13, France

$12{ }^{4}$ University of Paris Diderot, Sorbonne Paris Cité, 75250 Paris Cedex 13, France

$13{ }^{5}$ Département Hospitalo-Universitaire DHU PROTECT, Paris, France

$14{ }^{6}$ INSERM U1154, CNRS UMR 7196, Muséum National d'Histoire Naturelle, Paris, France

$15 *$ Equal contribution

17 Short title: Role of HSF2 in proteotoxicity

18 Key words: Bortezomib, cadherins, cell adhesion, cell survival, heat shock factor, proteotoxic 19 stress

21 " Corresponding author:

22 Lea Sistonen, Ph.D.

23 Faculty of Science and Engineering, Cell Biology

24 Åbo Akademi University

25 BioCity, Tykistökatu 6A

26 FIN-20520 Turku, Finland

27 Tel. +358-2-215-3311

28 Email: lea.sistonen@abo.fi 


\section{ABSTRACT}

31 Cellular ability to maintain proper protein homeostasis (proteostasis) is essential for survival upon 32 protein-damaging conditions. Heat shock transcription factor 2 (HSF2) is one of the human HSFs

33 activated in response to proteotoxic stress. HSF2 is dispensable for cell survival during acute heat 34 stress, but its amount and DNA-binding activity increase under prolonged proteotoxic stress conditions, such as proteasome inhibition. Nevertheless, the specific role(s) of HSF2 and the global HSF2-dependent gene expression profile during sustained stress have remained elusive. We found that HSF2 is required for cell survival during prolonged proteotoxicity, as shown by treating wild-

38 type and HSF2-deficient human osteosarcoma U2OS cells with the proteasome inhibitor

39 Bortezomib. Strikingly, our RNA-seq analyses revealed that HSF2 disruption leads to marked

40 downregulation of cadherin superfamily genes and subsequent functional impairment of cadherin-

41 mediated cell-cell adhesion. We propose HSF2 as a key regulator of genes belonging to the

42 cadherin superfamily. We also demonstrate that HSF2-dependent downregulation of cadherin-

43 mediated cell-cell adhesion predisposes U2OS cells to Bortezomib-induced proteotoxic stress. In

44 conclusion, we show that by maintaining cell-cell adhesion HSF2 is essential for cell survival and

45 thereby we describe a novel regime in the HSF-mediated protection against stress-induced protein 46 damage. 


\section{INTRODUCTION}

The cells in a human body are constantly exposed to environmental stressors, which challenge the maintenance of protein homeostasis (proteostasis). To survive insults that disturb proteostasis, cells rely on a selection of protective mechanisms that can be launched upon stress exposures. The heat shock response is a well-conserved stress protective pathway that is induced in response to cytosolic protein damage and mediated by heat shock transcription factors, HSFs [1]. Upon activation, HSFs oligomerize, accumulate in the nucleus, and bind to their target heat shock elements (HSEs) at multiple genomic loci [2-4]. The canonical HSF target genes encode molecular chaperones, such as heat shock proteins (HSPs), which assist in the maintenance of correct protein folding environment by refolding the misfolded proteins or directing them to protein degradation machineries [5]. In addition to regulating the transcriptional response to stress, HSFs are important in a variety of other physiological and pathological processes and the repertoire of HSF target genes extends greatly beyond the HSPs [6-10].

The human genome encodes six HSF family members (HSF1, HSF2, HSF4, HSF5, HSFX and HSFY), of which HSF1 and HSF2 are the most extensively studied [13]. Although HSF1 and HSF2 are homologous in their DNA-binding domains, they share only few similarities in the tissue expression patterns, regulatory mechanisms, and signals that stimulate their activity [1, 14]. HSF2 is an unstable protein and its expression is highly context-dependent, fluctuating in different cell and tissue types [15, 16], developmental stages [17, 18], and during the cell cycle [19]. Consequently, regulation of HSF2 protein levels has been generally accepted as the main determinant of its DNA-binding capacity [20, 21]. In mouse, lack of HSF2 results in developmental defects particularly associated with corticogenesis and spermatogenesis [22, 23]. HSF1 is essential for HSP expression and cell survival in a variety of acute stress conditions [13], whereas HSF2 is downregulated in cells exposed to acute heat stress [24] and has only a modulatory role in the regulation of HSP expression [4, 25, 26]. Interestingly, HSF2 is upregulated and acquires DNAbinding activity in cells exposed to proteasome inhibition induced by lactacystin or MG132 [20, $27,28]$.

The ubiquitin-proteasome system is one of the main cellular mechanisms regulating protein turnover, thereby affecting multiple aspects of cell physiology, such as signal transduction and apoptosis $[29,30]$. Due to the fundamental function in cell physiology, the proteasome complex has emerged as an important target for anti-cancer therapy [31]. The most common drug to inhibit proteasome function is Bortezomib (PS-341, VELCADE®), which is currently used as a standard 
81 treatment in hematological malignancies [32]. Bortezomib is a dipeptide boronic acid derivative

82 that targets the chymotrypsin-like activity of the $26 \mathrm{~S}$ proteasome, causing progressive 83 accumulation of damaged proteins [32-34]. By exposing human blood-derived primary cells to 84 clinically relevant concentrations of Bortezomib, Rossi and colleagues demonstrated that 85 prolonged proteasome inhibition results in a remarkable upregulation of HSF2 at both mRNA and protein levels [35]. That study also showed that HSF2, together with HSF1, localizes to the promoters of HSP70 and AIRAP (zinc finger AN1-type domain 2a), but that HSF2 is not required for the induction of these genes upon Bortezomib treatment $[35,36]$. Interestingly, proteasome inhibition has been shown to be severely toxic to HSF2-deficient mouse embryonic fibroblasts

90 [37], indicating that HSF2 is required for protection against proteotoxicity although its distinct role 91 and the global HSF2-dependent gene expression program have remained unknown.

In this study, by comparing Bortezomib-treated wild-type and HSF2 knock-out human osteosarcoma U2OS cells, we show that HSF2 is essential for cell survival upon prolonged Bortezomib-induced proteotoxicity. To our surprise, the RNA-seq analyses revealed that HSF2 disruption results in a profound downregulation of genes belonging to the cadherin superfamily and functional impairment of the cadherin-mediated cell-cell adhesion. Furthermore, we show that

98 inhibition of cadherin-mediated cell-cell adhesion predisposes human U2OS cells to Bortezomib-

99 induced cell death. Taken together, our results demonstrate that by maintaining cadherin-mediated

100 cell-cell adhesion, HSF2 acts as an important pro-survival factor during sustained proteotoxic 101 stress. 


\section{RESULTS}

\section{U2OS cells lacking HSF2 are predisposed to Bortezomib-induced proteotoxicity}

105 To explore the role of HSF2 during prolonged proteotoxic stress, we first examined the expression and cellular localization of HSF2 during Bortezomib (BTZ) treatment. Human osteosarcoma U2OS WT cells were treated with different concentrations of BTZ (0-100 nM) for 6 or $22 \mathrm{~h}$ and HSF2 protein levels were examined with immunoblotting. The time points were selected to assess both the short-term and the long-term exposure to BTZ. Interestingly, HSF2 was slightly upregulated

110 already at the 6-h time point, whereas at $22 \mathrm{~h}$ its expression was highly elevated (Fig. 1A). Indirect 111 immunofluorescence of U2OS WT cells revealed that HSF2, which is known to be both 112 cytoplasmic and nuclear [38, 39], resides in the nucleus already under control conditions and the 113 nuclear localization was further enhanced during BTZ treatment (Fig. 1B). These results are in line 114 with a previous report using human blood-derived primary cells [35] and show that also malignant 115 U2OS cells respond to BTZ treatment with marked increase in HSF2 levels and accumulation in 116 the nucleus.

118 Due to the prominent upregulation of HSF2 in cells treated with BTZ, we next asked if HSF2 is 119 required for the cellular survival under these stress conditions. We generated a U2OS HSF2 knockout cell line ( $2 \mathrm{KO}$ hereafter), where HSF2 expression was abolished by mutating the first exon of

121 the HSF2 gene using the CRISPR-Cas9 method. In these cells the protein expression of HSF2 was completely abrogated (Fig. 1C). U2OS WT and 2KO cells were treated with indicated concentrations of BTZ for $22 \mathrm{~h}$ and examined with microscopy. We observed a dramatic difference

124 in the viability of the WT and 2KO cells, since the cells lacking HSF2 exhibited an apoptotic non125 adherent phenotype in concentrations where WT cells remained adherent (Fig. 1D). Quantitative 126 cell viability measurements confirmed that the survival of $2 \mathrm{KO}$ cells was significantly impaired upon BTZ treatment (Fig. 1E). Furthermore, 2KO cells accumulated more cleaved PARP-1 than

128 WT cells, demonstrating a more pronounced activation of apoptosis [40] (Fig. 1F). Similar results were obtained with another HSF2 knock-out cell line (2KO\#2 hereafter) (Fig. S1A), confirming

130 that the observations are not specific for a single clone. In addition to BTZ, treatments with MG132,

131 a well-established proteasome inhibitor, and amino acid analogue L-canavanine, which causes 132 protein misfolding when incorporated into nascent peptide chains, clearly reduced viability of 133 HSF2-deficient cells (Fig. S1B-F). Previously, HSF2-null mouse embryonic fibroblasts $\left(H_{s} \mathrm{2}^{-/-}\right.$ 134 MEFs) have been shown to be sensitized to MG132 treatment as well as to sustained febrile-range 135 thermal stress [37, 41]. Altogether these results indicate that HSF2 is an important survival factor 136 in both murine and human cells when exposed to prolonged proteotoxic stress. 
138 To verify that the decreased survival of $2 \mathrm{KO}$ cells was not caused by off-target effects of the

139 CRISPR-Cas9 method, we transfected the U2OS WT cells with scramble (Scr) or HSF2-targeting 140 shRNA plasmids and treated the cells with BTZ for $22 \mathrm{~h}$. In accordance with the results obtained

141 with $2 \mathrm{KO}$ cells, transient HSF2 downregulation significantly reduced cell viability upon BTZ 142 treatment (Fig. 1G). Moreover, HSF2 downregulation enhanced the progression of apoptosis, 143 which was detected by increased accumulation of cleaved PARP-1 (Fig. 1H). Hence, we conclude 144 that HSF2 is required for cell survival upon BTZ-induced proteasome inhibition.

In contrast to HSF2, which has been found to be downregulated in a subset of human cancers [12], both the expression and activity of the closely related HSF1 are increased in a majority of studied cancer types [1]. A marker for HSF1 activation is phosphorylation of serine 326 (pS326) [7, 42], and HSF1 expression and pS326 were recently established as requirements for multiple myeloma cell survival during BTZ treatment [36]. Therefore, we examined whether the decreased survival of 2KO cells was due to impaired HSF1 expression or phosphorylation upon proteasome inhibition.

$152 \mathrm{U} 2 \mathrm{OS}$ WT and 2KO cells were treated with BTZ or MG132 and the HSF1 protein levels and S326 153 phosphorylation status were analyzed with immunoblotting. Interestingly, no difference in HSF1 154 expression or S326 phosphorylation between WT and 2KO cells was observed upon proteasome inhibition (Fig. S1G). These results demonstrate that although HSF1 is an essential survival factor during acute stress [1], it is not sufficient to protect cells during prolonged proteotoxicity.

\section{Bortezomib-induced heat shock response is not compromised in HSF2-deficient cells}

159 Similarly to many other surveillance transcription factors, such as p53 [43], HIF-1 $\alpha$ [44] and Nrf2 160 [45], HSF2 is an unstable protein under normal growth conditions [24]. HSF2 expression has been 161 shown to fluctuate in response to stress exposure, tumor progression, and during the cell cycle [12, $16219,24]$, and high expression levels of HSF2 correlate with its increased DNA-binding activity [20, 163 47]. Due to the massive increase in nuclear HSF2 levels upon BTZ treatment (Fig. 1B), we 164 investigated if the impaired survival of $2 \mathrm{KO}$ cells is caused by misregulation of HSF2 target genes. 165 U2OS WT and $2 \mathrm{KO}$ cells were treated with $25 \mathrm{nM} \mathrm{BTZ}$ for 6 or $10 \mathrm{~h}$ (Fig. 2A) and the global gene 166 expression profiles were analyzed with RNA-seq. The selected time points represent sub-lethal 167 proteotoxic stress conditions, at which the cell viability is not yet compromised (Fig. S2A). Before 168 mRNA purification, the knock-out phenotype was confirmed with immunoblotting (Fig. S2B). 169 Notably, stress-inducible hyperphosphorylation of HSF 1 [47] and increased HSP70 expression 170 were observed in both WT and 2KO cells (Fig. S2B). To identify the HSF2-dependent target genes, 
171 we first compared the inducible gene expression profiles between WT and 2KO cells in response

172 to $6 \mathrm{~h}$ and $10 \mathrm{~h} \mathrm{BTZ}$ treatment (Fig. 2A). Differentially expressed (DE) genes were determined

173 with the Bioconductor R package Limma [48], with fold change $\geq 3$ and false discovery rate (FDR)

$174<0.001$, from quadruplet samples that correlated well to each other (Fig. S2C). According to the

175 analysis, BTZ treatment resulted in a significant upregulation and downregulation of genes in WT

176 (>600 and $>300$, respectively) and $2 \mathrm{KO}$ (> $>500$ and $>200$, respectively) cells (Fig. 2B, Table S1).

177 The complete dataset is available at Gene Expression Omnibus under accession number:

178 GSE115973.

180 The HSF-regulated heat shock response is one of the main cellular survival pathways induced by 181 proteotoxic stress and it is characterized by simultaneous upregulation of multiple genes essential 182 for maintaining the correct protein folding environment $[1,4]$. To examine whether the heat shock 183 response is compromised in $2 \mathrm{KO}$ cells, we analyzed the inducible expression patterns of all human 184 molecular chaperone genes [49], in WT and 2KO cells treated with BTZ. Intriguingly, the 185 chaperone expression profiles of WT and $2 \mathrm{KO}$ cells were nearly identical, and only $H S P B 2$, 186 DNAJC1,2 and DNAJC18 exhibited clearly distinct expression patterns in 2KO cells (Fig. 2C). A 187 closer examination of the RNA-seq data for the expression of selected chaperone genes, i.e. 188 HSPA1A (HSP70), HSP90AAl (HSP90), HSPA6 (HSP70B'), and HSPB1 (HSP27), revealed equal 189 or even higher expression levels in 2KO cells than in WT cells (Fig. 2D). HSF2 has been previously 190 shown to negatively modulate HSF1-mediated activation of specific HSP targets [19, 25, 26], 191 which likely explains the higher expression of HSPBI and HSP90AA1 in 2KO cells upon BTZ 192 treatment (Fig. 2D). In response to proteotoxic stress, HSF2 localizes also to the promoters of 193 HSP90 co-chaperone and polyubiquitin genes [2]. To study whether the regulation of these genes 194 was disturbed in 2KO cells, HSP90 co-chaperones PTGES3 (p23) and AHSA1 (AHA1) as well as 195 polyubiquitin genes ( $U B B$ and $U B C$ ) were examined from our RNA-seq data. Since no significant 196 differences were observed in the expression patterns of any of these genes (Fig. 2E), we conclude 197 that lack of HSF2 does not compromise the induction of the heat shock response upon BTZ 198 treatment.

\section{Disruption of HSF2 leads to misregulation of cell adhesion associated genes}

201 To determine the differences between the WT and $2 \mathrm{KO}$ cells, we examined the DE genes in $2 \mathrm{KO}$ 202 : WT comparison pair at each experimental time point (control, 6, and $10 \mathrm{~h}$ ) (Fig. 3A). Despite the 203 stringent cutoff criteria (FC $\geq 3$, FDR 0.001), 2KO cells exhibited significant misregulation of 819 204 genes already under normal growth conditions (2KO C : WT C), and the proportion of upregulated 
and downregulated genes remained similar throughout the BTZ treatments $(2 \mathrm{KO} 6 \mathrm{~h}: \mathrm{WT} 6 \mathrm{~h}$, $2 \mathrm{KO} 10 \mathrm{~h}: 2 \mathrm{KO} 10 \mathrm{~h}$ ) (Fig. 3B, Table S1). GO term analysis of the misregulated genes revealed a specific enrichment of terms related to cell adhesion and cell-cell adhesion via plasma membrane adhesion molecules (Fig. 3C, Table S1). Similar GO terms among the comparison pairs implied that the genes misregulated in $2 \mathrm{KO}$ cells are tightly linked to cellular adhesion properties both under control and stress conditions (Fig. 3C).

212 To identify the adhesion molecules that are abnormally expressed in $2 \mathrm{KO}$ cells in both control and 213 stress conditions, the gene set overlaps were examined with Venn diagrams. Among the 214 comparison pairs, a total of 114 and 277 genes were upregulated and downregulated, respectively

215 (Fig. 3D). Functional cluster annotation of the 114 upregulated genes with the DAVID analysis 216 tool [50] confirmed the strong association to cell adhesion and extracellular matrix, including 217 collagens (COL16A1, COL18A1) and laminins (LAMB1 and LAMA5) (Fig. 3E left panel and S3).

218 Interestingly, the 277 downregulated genes included members from many cadherin sub-families, 219 for example protocadherins (PCDHA1 and PCDHA7), desmosomal cadherins (DSC2), and Fat220 Dachsous cadherins (FAT2), suggesting that the cadherin-mediated cell adhesion is extensively 221 misregulated in $2 \mathrm{KO}$ cells (Fig. 3E, right panel). Cadherins are transmembrane adhesion molecules 222 that mediate $\mathrm{Ca}^{2+}$-dependent cell-cell adhesion via the conserved extracellular cadherin domains 223 [51]. The human genome encodes 110 cadherin genes, which together form the cadherin 224 superfamily consisting of distinct cadherin sub-families [51]. The most prominent changes were 225 detected in protocadherins, as 13 distinct protocadherin genes were significantly downregulated in $2262 \mathrm{KO}$ cells grown either under normal growth conditions or exposed to BTZ-induced stress (Fig. $2273 \mathrm{E})$.

\section{Cells lacking HSF2 display abnormal cadherin expression}

230 Since the cadherin genes appeared as a novel HSF2-dependent gene group and showed significant 231 misregulation in multiple sub-family members, we examined the expression profiles of all cadherin 232 superfamily genes in $2 \mathrm{KO}$ cells. Normalized gene expression data were used to generate a heat 233 map encompassing all the cadherin superfamily genes encoded by the human genome. By 234 comparing the expression profiles of WT and 2KO cells in control and BTZ-induced stress 235 conditions, we observed a clear downregulation of the entire cadherin superfamily, as at least one member from every sub-family was found downregulated in $2 \mathrm{KO}$ cells. These included classical

237 cadherins ( $C D H 2$ and CDH6), desmosomal cadherins (DSC2 and DSG2), CDH23-PCDH15 238 cadherins (CDH12), Fat-Dachsous cadherins (FAT2 and FAT4), Flamingo cadherins (CELSRI), 
and Calsyntenins (CLSTN2) (Fig. 4A, Table S1). The most striking downregulation was detected in clustered $\alpha$-, $\beta$-, and $\gamma$-protocadherins [52], of which $92 \%$ were found abnormally expressed in $2 \mathrm{KO}$ cells (Fig. 4A). Based on these results, we propose that the members of the cadherin superfamily are the main adhesion molecules downregulated in HSF2-depleted U2OS cells.

For understanding the biological relevance of the RNA-seq analyses, we next determined the protein expression levels of $\mathrm{N}$-cadherin $(\mathrm{CDH} 2)$ and clustered $\gamma$-protocadherins (PCDH $\gamma \mathrm{A})$ by immunoblotting. As shown in Figure 4B, both $\mathrm{N}$-cadherin and $\gamma$-protocadherins were found markedly downregulated also at the protein level in control and BTZ-treated 2KO cells (Fig. 4B). Since cadherins are essential in mediating $\mathrm{Ca}^{2+}$-dependent cell-cell contacts, we examined the functional impact of our observations with cell aggregation assay, where single cells are allowed to freely make cell-cell adhesion contacts in suspension. U2OS WT and 2KO cells were suspended in cell aggregation buffer supplemented with either $\mathrm{CaCl}_{2}$ or EDTA. WT cells supplemented with $\mathrm{Ca}^{2+}$ formed large cell aggregates, which were completely abolished in $\mathrm{Ca}^{2+}$-chelating conditions (EDTA) (Fig. 4C). In stark contrast, 2KO cells were unable to form cell aggregates even in the presence of $\mathrm{Ca}^{2+}$ (Fig. 4C), indicating that HSF2 is required to maintain $\mathrm{Ca}^{2+}$-dependent cell-cell contacts. Loss of distinct cell-cell adhesion molecules has been associated with cellular inability to form three-dimensional (3D) spheroids in ultra-low attachment round bottom plates [53]. We explored the spheroid forming capacity of U2OS WT and $2 \mathrm{KO}$ cells by culturing the cells in 3D extracellular matrix (ECM) using Matrigel. As expected, the spheroids originating from $2 \mathrm{KO}$ cells were significantly smaller than the WT spheroids (Fig. 4D), further confirming the functional impairment of cell-cell adhesion of these cells. A profound decline in the expression and function of cadherin superfamily proteins was observed also in 2KO\#2 cells (Fig. S4), demonstrating that the alterations are not specific for a single cell clone. Altogether these results show, for the first time, that lack of HSF2 leads to disrupted cadherin expression at mRNA and protein levels, and results in functional deterioration of cadherin-mediated cell-cell adhesion.

\section{Impaired cell-cell adhesion sensitizes cells to proteotoxic stress}

Albeit it is well-acknowledged that cadherins are essential mediators of tissue integrity and pivotal in regulating the development of multicellular organisms [51, 52], their impact on stress resistance has remained largely unexplored. To examine whether the observed impairment of cell-cell adhesion in $2 \mathrm{KO}$ cells can also contribute to the susceptibility of the cells to BTZ-induced stress,

271 we aimed at restoring the cellular adhesion properties by re-introducing N-cadherin to $2 \mathrm{KO}$ cells.

272 For this purpose, WT and 2KO cells were transfected with either Mock or N-cadherin plasmids 
273 and the N-cadherin expression was examined with immunoblotting (Fig. 5A). As shown in Figure $2745 \mathrm{~A}$, we were able to restore the $\mathrm{N}$-cadherin levels in $2 \mathrm{KO}$ cells. Moreover, re-introduction of $\mathrm{N}$ 275 cadherin resulted in a functional rescue of cell-cell adhesion in $2 \mathrm{KO}$ cells (Fig. 5B). Importantly, 276 when exposed to BTZ, the $2 \mathrm{KO}$ cells expressing exogenous $\mathrm{N}$-cadherin displayed significantly less 277 cleaved PARP-1 than the Mock-transfected cells (Fig. 5C), suggesting that restoration of cell-cell adhesion can suppress cell death upon BTZ-induced proteotoxic stress.

280 All cadherin superfamily proteins are characterized by extracellular cadherin repeat domains, 281 which mediate homophilic adhesion contacts between adjacent cells [54]. Stabilization of the 282 extracellular domains is regulated by $\mathrm{Ca}^{2+}$, which binds to the interdomain regions of consecutive 283 cadherin repeats and rigidifies the ectodomain structure. To be able to comprehensively investigate 284 the role of cadherins in the cellular resistance to proteotoxic stress, we first treated the WT and $2852 \mathrm{KO}$ cells with BTZ for $20 \mathrm{~h}$ to induce proteotoxic stress, after which the whole cadherin-mediated 286 cell-cell adhesion program was destabilized by depleting the extracellular $\mathrm{Ca}^{2+}$ with 3 mM EDTA 287 (Fig. 5D). Serum-free culture conditions were used to enable full depletion of extracellular $\mathrm{Ca}^{2+}$. 288 Intriguingly, we observed that $\mathrm{Ca}^{2+}$-depletion intensified cell death, which was evidenced by the 289 significantly reduced WT cell survival after treatment with both BTZ and EDTA (Fig. 5D). In 290 contrast, the viability of $2 \mathrm{KO}$ cells was not markedly affected by BTZ treatment combined with $291 \mathrm{Ca}^{2+}$-depletion when compared to the BTZ treatment alone (Fig. 5D). Altogether, these results 292 show that cadherin-mediated cell-cell adhesion is a key determinant of cell survival upon BTZ 293 treatment and that destabilization of cadherin contacts predisposes U2OS cells to prolonged 294 proteotoxic stress. 


\section{DISCUSSION}

Maintenance of cellular protein homeostasis (proteostasis) is crucial for the viability of all cells and organisms [13]. The heat shock response is critical for promoting proteostasis and it is under strict control of the heat shock transcription factors, HSFs. Until now, the comprehensive role of HSF2 in the cellular response to sustained proteotoxicity has remained unknown. We hypothesized that HSF2 is required for protecting cells against progressive accumulation of protein damage. We used the clinically relevant proteasome inhibitor Bortezomib (BTZ, PS-341, VELCADE®) as our experimental tool to induce long-term proteotoxic stress. BTZ treatment has been previously shown to upregulate HSF2 at both mRNA and protein levels in blood-derived human primary cells and to induce HSF2 binding at specific gene loci [35]. Our data confirmed the earlier results by showing that BTZ treatment leads to a remarkable increase in HSF2 protein levels also in malignant human cells. Moreover, we demonstrate that the amount of nuclear HSF2 is highly increased in BTZtreated cells, strongly suggesting that HSF2 specifically responds to proteasome inhibition. We found that HSF2 is not only activated by BTZ-induced proteotoxicity, but it is absolutely essential

310 for cell survival under these conditions. Based on our results, together with the reduced viability 311 of $\mathrm{Hsf}_{\mathrm{s}} \mathrm{-}^{-/}$MEFs treated with MG132 or exposed to febrile-range thermal stress [37, 41], we conclude that HSF2 is required to protect cells against progressive accumulation of damaged

\section{3 proteins.}

315 Elevated protein levels of HSF1 and its phosphorylation on serine 326 were recently shown to be 316 a prerequisite for multiple myeloma cell survival upon BTZ treatment $[36,55]$. Therefore, we 317 explored whether HSF2 depletion sensitizes cells to BTZ through misregulated HSF1, specifically, 318 or the heat shock response in general. Neither difference in HSF1 levels nor serine 326 319 phosphorylation was detected between WT and HSF2-depleted cells treated with proteasome inhibitors. Moreover, the classical heat shock response, as characterized by the global upregulation of molecular chaperones, HSP90 co-chaperones, and polyubiquitin genes, was not compromised in cells lacking HSF2. These results indicate that HSF2 can promote cell survival independently of HSF1.

Previously, HSF2 has been shown to have only a modest effect on gene expression [2, 13, 41]. To our surprise, despite the stringent cutoff criteria ( $F C \geq 3$, FDR 0.001), we found a great number of genes displaying altered expression profiles in cells lacking HSF2. Among the most prominently

328 misregulated genes were those belonging to the cadherin superfamily. Cadherins are a large group 329 of transmembrane adhesion molecules, which mediate $\mathrm{Ca}^{2+}$-dependent cell-cell adhesion and 
330 thereby function as essential mediators of tissue integrity [51]. Lack of HSF2 led to a profound

331 downregulation of cadherins both at mRNA and protein levels as well as functional impairment of

332 cadherin-mediated cell-cell adhesion contacts. Our finding of abnormal cadherin expression

333 already under normal growth conditions, together with the earlier results on HSF2 displaying DNA-

334 binding capacity in the absence of stress [2, 15, 56], suggests that HSF2 has a physiological role in

335 regulating cadherin gene expression. Excitingly, impaired migration and mispositioning of neurons

336 have been shown to underlie the corticogenesis defects in $H_{s f} 2^{-/}$mice $[22,23]$, and cadherin

337 superfamily proteins are fundamental for correct neuronal migration [57]. Thus, it is tempting to

338 speculate that the HSF2-dependent disruption of cadherin-mediated cell-cell contacts contributes

339 to the abnormal corticogenesis of $\mathrm{Hsf}^{-/-}$mice.

341 The downregulation of cadherin gene expression raises important questions about the mechanisms

342 by which HSF2 regulates these genes. Genome-wide mapping of HSF2 binding sites has been

343 previously determined with ChIP-seq in human K562 erythroleukemia cells [2] and in mouse testis

344 [58]. Remarkably, both studies identified HSF2 occupancy on multiple cadherin superfamily 345 genes. Since non-adherent K562 cells are deficient of endogenously expressed classical cadherins 346 and distinct protocadherins [59], it is not surprising that HSF2 was found to occupy only the CLSTN 347 gene under control growth conditions [2]. However, upon acute heat stress, HSF2 binding was 348 observed at classical cadherins (CDH4), desmogleins (DSG2), Fat-Dachous cadherins (DCHS2), 349 Flamingo cadherins (CELSR2), and CDH23-PCDH15 cadherins (CDH23) [2], demonstrating that 350 multiple genes belonging to the cadherin superfamily can be targeted by HSF2 in human cells. In 351 mouse testis, HSF2 was also shown to occupy several cadherin genes, including $C D H 15, C D H 5$, $352 \mathrm{CDH18}, \mathrm{CDH13}, \mathrm{FAT1}, \mathrm{PCDH}$, $\mathrm{PCDH17}$, and PCDHA1 [58]. Altogether these results suggest 353 that HSF2 is a key regulator of cadherin genes. Future studies are warranted for comprehensive 354 analyses of direct HSF2 targets in cells and tissues across a variety of model systems.

356 Failure in maintenance of proteostasis is a hallmark of aging and neurodegenerative diseases [60]. 357 Intriguingly, in a mouse model of Huntington's disease, lack of HSF2 was shown to predispose 358 mouse brain to poly-Q aggregates and reduce life span [41], suggesting that HSF2 is required to 359 protect neurons from progressive accumulation of damaged proteins. Cell survival upon 360 proteotoxic stress has been conventionally considered to depend on inducible transcriptional 361 programs, such as the heat shock response or the unfolded protein response [1, 61]. However, in 362 this study, we show that HSF2-dependent maintenance of cell-cell adhesion is an essential and 363 novel determinant of proteotoxic stress resistance. Our results indicate that misregulation of distinct 
364 cellular properties already under normal growth conditions can sensitize cells to proteotoxicity. 365 HSF1 and HSF2 might represent the two arms of the cellular resistance towards proteotoxic stress;

366 HSF1 as an acute responder to protein damage and HSF2 as a factor maintaining the long-term 367 stress resistance. Notably, a meta-analysis of transcriptional changes associated with Alzheimer's 368 disease and aging, HSF2 was identified as a gene commonly downregulated during aging [62]. 369 Thus, it is possible that the age-associated downregulation of HSF2 and subsequent disruption of 370 cadherin-mediated cell-cell adhesion, participates in sensitizing cells, such as neurons, to aggregate 371 mismanagement. 


\section{MATERIALS AND METHODS}

\section{CRISPR-Cas9}

375 Guide RNAs (gRNA) targeting the exon 1 of HSF2 were designed using CRISPOR software 376 (http://crispor.tefor.net/) and cloned into pMLM3636 gRNA expression plasmid (a gift from Keith 377 Joung, Addgene plasmid \#43860). U2OS cells were transfected with Cas9 and gRNA expression 378 plasmids using Amaxa electroporation as recommended by the manufacturer (Lonza). The hCas9 plasmid was a gift from George Church (Addgene plasmid \#41815). One week after transfections, cells were seeded at single cell density. Clones were genotyped by DNA sequencing of PCR products spanning the targeted region of the HSF2 gene. The selected U2OS clones presented 3

382 different outframe mutations on HSF2 exon 1, each corresponding to a different allele (Table S2).

383 Guide RNA sequence targeting the $1^{\text {st }}$ AUG of the HSF2 exon 1: 5'UGCGCCGCGUUAACAAUGAA-3'. Following primers were used for PCR for validation: HSF2 knock-out cell clone 1 (hereafter 2KO) and HSF2 knock-out cell clone 2 (hereafter 2KO\#2).

\section{Cell culture and treatments}

390 Cells were cultured in DMEM (Dulbecco's Modified Eagle's media, D6171, Sigma-Aldrich), supplemented with $10 \%$ fetal calf serum, $2 \mathrm{mM}$ L-glutamine and $100 \mu \mathrm{g} / \mathrm{ml}$ penicillinstreptomycin, and grown in $5 \% \mathrm{CO}_{2}$ at $37^{\circ} \mathrm{C}$. Proteasome inhibition was induced with Bortezomib

393 (BTZ, sc-217785, Santa Cruz Biotechnology) or MG132 (Z-Leu-Leu-Leu-H, 317-V, Peptide 394 Institute Inc.). Both inhibitors were diluted in DMSO (dimethyl sulfoxide, D8418, Sigma-Aldrich) and applied to cells in final concentrations indicated in the figures. Control cells were treated with DMSO only.

To induce protein misfolding with amino acid analogues, cells were starved for $17 \mathrm{~h}$ in L-arginine free culture medium (A14431-01, Gibco) supplemented with $10 \%$ fetal calf serum, 2 mM L-

400 glutamine and $100 \mu \mathrm{g} / \mathrm{ml}$ penicillin-streptomycin. Following that, L-Canavanine sulfate salt 401 (C9758, Sigma) was applied to the cells in final concentrations indicated in the figure. Cells were 402 treated for 3 or $6 \mathrm{~h}$. After the treatments, cells were visualized with Leica phase contrast 403 microscope, an EVOS FL Cell Imaging System (Thermo Fisher Scientific), or an Axio Vert A1404 FL LED microscope (Carl Zeiss) and harvested for further analyses.

\section{Transfections}


For transfections, 6 x $10^{6}$ U2OS WT or 2KO cells were suspended in $400 \mu$ l of Opti-MEM (11058021, Gibco) and subjected to electroporation $(230 \mathrm{~V}, 975 \mu \mathrm{F})$ in BTX electroporation cuvettes (450126, BTX). To downregulate HSF2 in WT cells HSF2 targeting shRNA and Scr vectors (previously described in [25]) were used. For restoring the protein levels of N-Cadherin in 2KO cells, a N-cadherin in pCCL-c-MNDU3c-PGK-EGFP vector co-expressing EGFP (a gift from Nora Heisterkamp, Addgene plasmid \#38153) and pcDNA3.1/myc-His(-)A (used as Mock) were used. One day after transfection, cells were trypsinized, counted, re-plated, and let to recover for $24 \mathrm{~h}$ before BTZ treatments.

For cell aggregation assays, cells were transfected with GenJet (\#SL100489-OS, SignaGen Laboratories) according to manufacturer's instructions. Briefly, cells were plated 18 to $24 \mathrm{~h}$ prior to transfections to ensure $80 \%$ confluency, and fresh culture media with supplements was added to the cells before transfections. The N-Cadherin encoding vector (described above) was used for transfections, and pEGFP-N1 (clonetech) was used as a Mock. The plasmids and the GenJet reagent were diluted in serum free media, and applied to the cells in a ratio of 1:2 (DNA:GenJet reagent). Cells were incubated with the DNA:GenJet mixture for $4 \mathrm{~h}$, washed with PBS, and supplemented with complete culture media. Cells were let to recover for $24 \mathrm{~h}$ before the cell aggregation experiments.

\section{Immunoblotting}

Cells were collected in culture media, washed with PBS (L0615, BioWest) and lysed in lysis buffer [50 mM HEPES, pH 7.4, 150 mM NaCl, 1 mM EDTA, 2 mM MgCl $2,1 \%$ Triton X-100, $10 \%$ glycerol, 1 x complete ${ }^{\mathrm{TM}}$ Protease Inhibitor Cocktail (04693159001, Roche Diagnostics), $50 \mathrm{mM}$ $\mathrm{NaF}, 0.2 \mathrm{mM} \mathrm{Na}_{3} \mathrm{VO}_{4}$ ]. Protein concentration of the lysates was determined with Bradford assay. Equal amounts of cell lysates were resolved on 4-20\% or 7.5 \% Mini-PROTEAN ${ }^{\circledR}$ TGX precast gels (Bio-Rad) and the proteins were transferred to a nitrocellulose membrane. For HSF2 detection, membranes were boiled for $15 \mathrm{~min}$ in $\mathrm{MQ}-\mathrm{H}_{2} \mathrm{O}$ and blocked in $3 \%$ milk-PBS-Tween20 solution for $1 \mathrm{~h}$ at RT. Primary antibodies were diluted in $0.5 \% \mathrm{BSA}-\mathrm{PBS}-0.02 \% \mathrm{NaN}_{3}$ and the membranes were incubated in respective primary antibodies overnight at $4{ }^{\circ} \mathrm{C}$. The following antibodies were used: anti-GAPDH (ab9485, Abcam), anti-HSC70 (ADI-SPA-815, Enzo Life Sciences), anti-HSF1 (ADI-SPA-901, Enzo Life Sciences), anti-HSF1 pS326 (ab76076, Abcam), anti-HSF2 (HPA031455, Sigma-Aldrich), anti-HSP70 (ADI-SPA-810, Enzo Life Sciences), N-cadherin (041126, Millipore or ab76057, Abcam), anti-PARP-1 (F-2, sc-8007, Santa Cruz Biotechnology), antiPanPCDH $\gamma \mathrm{A}$ (75-178, NeuroMab), and anti- $\beta$-Tubulin (T8328, Sigma-Aldrich). Secondary 
antibodies were HRP-conjugated and purchased from Promega, GE Healthcare or Abcam. All immunoblotting experiments were performed at least three times.

\section{Immunofluorescence}

$2 \times 10^{5}$ U2OS WT cells were plated on coverslips or MatTek plates (P35GC-.5-14-C, MatTek Corporation) $24 \mathrm{~h}$ before treatments. Cells were fixed with $4 \%$ paraformaldehyde (PFA) for 15 min, permeabilized in $0.1 \%$ Triton-X-100 in PBS and washed three times with PBST (PBS-0.5\% Tween20). Cells were blocked with $10 \%$ FBS in PBS for $1 \mathrm{~h}$ at room temperature and incubated overnight at $4{ }^{\circ} \mathrm{C}$ with a primary anti-HSF2 antibody (HPA031455, Sigma-Aldrich), which was diluted 1:20 in 10\% FBS-PBS. Secondary goat anti-rabbit Alexa Fluor 488 (R37116, Invitrogen) was diluted 1:500 in 10\% FBS-PBS and the cells were incubated for $1 \mathrm{~h}$ in RT. Cells were washed three times with PBST, incubated with 300 nM DAPI diluted in PBS or mounted in MowiolDABCO mounting medium, and imaged with a $3 \mathrm{i}$ CSU-W1 spinning disc confocal microscope (Intelligent Imaging Innovations).

\section{Calcein assays}

$5 \times 10^{3}$ U2OS WT and HSF2 KO cells were cultured in clear bottom 96-well plate (6005181, Perking Elmer) in complete culture media. Cells were treated with indicated concentrations of Bortezomib or MG132 for $22 \mathrm{~h}$. For calcium-depletion, cells were treated with or without $25 \mathrm{nM}$ Bortezomib for $20 \mathrm{~h}$ in serum free media. The extracellular calcium was depleted with EDTA in calcium free media and the Bortezomib treatment was continued for $2 \mathrm{~h}$. Control cells were treated with DMSO. After treatments cells were washed with PBS and incubated for 30 min at $37^{\circ} \mathrm{C}$ with Calcein AM (4892-010-K, R\&D Systems) diluted 1:1000 in PBS. Fluorescence intensity was measured with Hidex Sense microplate reader (HIDEX Corp) with excitation and emission wavelengths $485 \mathrm{~nm}$ and $535 \mathrm{~nm}$, respectively. Respective blank values were subtracted from the sample values and the viability of untreated control samples (WT or 2KO) was set to value 1. All the measurements were repeated at least three times.

\section{Cell aggregation assays}

After trypsinization, $5 \times 10^{5} \mathrm{U} 2 \mathrm{OS}$ WT and $2 \mathrm{KO}$ cells were suspended in $2 \mathrm{ml}$ of aggregation assay buffer $\left(137 \mathrm{mM} \mathrm{NaCl}, 5.4 \mathrm{mM} \mathrm{KCl}, 0.63 \mathrm{mM}, \mathrm{Na}_{2} \mathrm{HPO}_{4}, 5.5 \mathrm{mM}\right.$ glucose, and $10 \mathrm{mM}$ HEPES, $\mathrm{pH}$ 7.4) supplemented with either $3 \mathrm{mM} \mathrm{CaCl}_{2}$ or $3 \mathrm{mM}$ EDTA. Cells were rotated for $2.5 \mathrm{~h}$ in 150 rpm at $37^{\circ} \mathrm{C}$, after which the aggregates were imaged with the EVOS FL Cell Imaging System (Thermo Fisher Scientific) or with an Axio Vert A1-FL LED microscope (Carl Zeiss). Cell 
aggregation assays were performed in biological triplicates. The area of the three biggest aggregates in each sample was measured with ImageJ (U. S. National Institutes of Health, Bethesda, Maryland, USA) for quantification purposes. All cell aggregation experiments were repeated at least three times.

\section{RNA-sequencing}

$2 \times 10^{6}$ U2OS WT and HSF2 KO cells were plated and cultured overnight. Following day, cells were treated with $25 \mathrm{nM}$ BTZ for 6 or $10 \mathrm{~h}$. Control cells were treated with DMSO. Cells were collected, and total RNA was purified with AllPrep DNA/RNA/miRNA Universal Kit (80224, Qiagen) according to manufacturer's instructions. Genomic DNA from mRNA columns was digested with DNAse I. The RNA library was prepared according to Illumina TruSeq ${ }^{\circledR}$ Stranded mRNA Sample Preparation Guide (part \#15031047). Briefly, poly-A containing mRNA molecules were purified with poly-T oligo magnetic beads and fragmented with divalent cations under elevated temperatures. For first-strand cDNA synthesis, RNA fragments were copied using reverse transcriptase and random primers. Unique Illumina TrueSeq indexing adapters were ligated to each sample. The quality and concentration of cDNA samples were analyzed with Advanced Analytical Fragment Analyzer and Bioanalyzer 2100 (Agilent, Santa Clara, CA, USA) and Qubit ${ }^{\circledR}$ Fluorometric Quantitation (Life Technologies). Samples were sequenced with Illumina HiSeq 3000 (Illumina). All the experimental steps after the RNA extraction were conducted in the Finnish Microarray and Sequencing Center, Turku, Finland. RNA-sequencing was performed from four independent sample series.

\section{Bioinformatic analysis}

The quality of the raw sequencing reads was confirmed with FastQC version 0.20.1 and aligned against the hg38 human genome assembly using TopHat2 version 2.1.0. Subreads version 1.5.0 was used to calculate gene level expression counts according to RefSeq-based gene annotations. The downstream analysis was carried out with $\mathrm{R}$ and Bioconductor. The data was normalized with TMM normalization method on the edgeR package. In all sample groups, the Spearman's correlation value was above 0.97 , indicating high reproducibility. Statistical testing between the sample groups was carried out using Bioconductor R package Limma [48] and the differentially expressed genes were filtered using fold change $\geq 3$ and false discovery rate (FDR) of 0.001 as cutoff. Enrichment analysis for the differentially expressed (DE) filtered genes was performed with topGO and GOstats packages. GO terms in each comparison pair were ranked according to their significance (lowest p-value) and the most significantly changed terms were selected for the 
509 figures. Additional information regarding the term IDs can be found from

510 http://www.geneontology.org.

\section{Quantitative RT-PCR (qRT-PCR)}

513 RNA was isolated using a RNeasy mini kit (74106, Qiagen) according to the manufacturer's 514 instructions and quantified using a NanoDrop ND-1000 spectrophotometer (Thermo Fisher 515 Scientific). Following that, $1 \mu \mathrm{g}$ of total RNA was reverse transcribed with an iScript ${ }^{\mathrm{TM}}$ cDNA 516 Synthesis Kit (\#1708891, Bio-Rad). SensiFAST ${ }^{\text {тм }}$ Probe Lo-ROX and SensiFAST ${ }^{\text {тм }}$ SYBR ${ }^{\circledR}$ Lo517 ROX kits (Bioline) were used for qRT-PCRs that were performed with QuantStudio 3 Real-Time 518 PCR system (Applied Biosystems, Thermo Fisher Scientific). All primers and probes were 519 purchased from Sigma Aldrich. The following forward (f) and reverse (r) primers, and probes (pr) 520 were used: fRNA18S5, 5'-GCAATTATTCCCCATGAACG-3'; rRNA18S, 521 5'-GGGACTTAATCAACGCAAGC-3'; prRNA18S5, 5'-FAM-TTCCCAGTAAGTGCG 522 GGTC-BHQ-3'; fDSC2; 5'-ATCCATTAGAGGACACACTCTGA-3'; 523 5'-GCCACCGATCCTCTTCCTTC-3'; fPCDHA6, 5'-TGACTGTTGAATGATGGCGGA-3'; 524 rPCDHA6, 5'-TCGGGTACGGAGTAGTGGAG-3'; fPCDHA10, 5'- AGGCATCAGCCAGTTTCTCAA-3'; rPCDHA10, 5'-GAGAGCAGCAGACACTGGAC-3' .

526 The mRNA expression levels were normalized against the respective 18S RNA (RNA18S5) 527 expression in a given sample. All reactions were run in triplicate from samples derived from four 528 biological replicates.

\section{D cell culture and immunofluorescence}

531 For examining cell growth in 3D, cells were embedded in growth factor reduced Matrigel 532 (\#356231, Corning) and cultured in Angiogenesis $\mu$-slides (\#81501, Ibidi) as described previously 533 [63]. Briefly, wells were filled with $10 \mu 1$ of Matrigel:culture medium (1:1 ratio), which was 534 polymerized at $37^{\circ} \mathrm{C}$ for $60 \mathrm{~min}$. WT, $2 \mathrm{KO}$, or $2 \mathrm{KO} \# 2$ cells were seeded on top of the gel at a 535 density of 700 cells per well, let to attach at $37^{\circ} \mathrm{C}$ for $2 \mathrm{~h}$, and covered with $20 \mu 1$ of Matrigel:culture 536 medium (1:4 ratio). The upper layer of Matrigel:culture medium was polymerized at $37{ }^{\circ} \mathrm{C}$ 537 overnight, and appropriate humidity was ensured by adding droplets of $\mathrm{MQ}-\mathrm{H}_{2} \mathrm{O}$ between the 538 wells. Culture medium was changed every second day, and cell growth was monitored by imaging the cultures with a Zeiss Axio Vert A1-FL LED microscope (Carl Zeiss).

541 For immunofluorescence, spheroids were washed with $40 \mu 1$ of PBS and fixed with $25 \mu 1$ of $4 \%$ 542 PFA for $20 \mathrm{~min}$ at room temperature, followed by three washes with $40 \mu \mathrm{l}$ of PBS. Spheroids were 
543 stained with $25 \mu 1$ of $0.7 \%$ Triton X-100, 1:500 Alexa Fluor 488 Phalloidin (\#A12379, \#A22287,

544 Thermo Fisher Scientific), $300 \mathrm{nM}$ DAPI in PBS at room temperature for $1 \mathrm{~h}$. The stained spheroids

545 were stored in PBS at $4{ }^{\circ} \mathrm{C}$ until imaging.

547 The spheroids were imaged as z-stacks with a $3 \mathrm{i}$ CSU-W1 spinning disc confocal microscope

548 (Intelligent Imaging Innovations) using the same settings between the repeats. Spheroid volume

549 was calculated based on the phalloidin staining using ImageJ v1.51n [64] software with the 3D

550 Object Counter v2.0 (Bolte and Cordelieres, 2006) plugin. The threshold for background and object

551 voxels was manually adjusted for each image in order to capture the whole volume of each

552 spheroid.

\section{Visualization of the data}

555 Heatmaps were generated with GraphPad Prism 7 Software (GraphPad Prism Software, La Jolla 556 California USA, http://www.graphpad.com). Venn diagrams were generated with BioVenn web 557 application (http://www.cmbi.ru.nl/cdd/biovenn/). DAVID Bioinformatic Resources 6.7 558 (https://david-d.ncifcrf.gov/home.jsp) was used for functional annotation clustering.

\section{Statistical analysis}

561 All statistical analyses were performed with GraphPad Prism 7 Software (GraphPad Prism 562 Software, La Jolla California USA, http://www.graphpad.com). The statistical significance was 563 analyzed with two-way ANOVA and Holm-Sidak's post-hoc test unless indicated differently. 


\section{ACKNOWLEDGEMENTS}

566 We are grateful to Joshua Weiner (University of Iowa, Iowa, US) for helpful discussions and advice

567 and for providing us with the anti-PanPCDH $\gamma \mathrm{A}$ antibody. All members of the Sistonen laboratory and Véronique Dubreuil from the Mezger laboratory are thanked for their valuable comments and critical review of the manuscript. Imaging was performed at the Cell Imaging Core, Turku Centre for Biotechnology, University of Turku and Åbo Akademi University. The instruments used in this project belong to the infrastructure of Biocenter Finland. We especially thank Markku Saari and Jouko Sandholm from the Cell Imaging Core of Turku Centre for Biotechnology for technical assistance and advice. The Bioinformatics unit of Turku Centre for Biotechnology is acknowledged for their expert assistance with the RNA-sequencing data analysis. The Bioinformatics unit is supported by University of Turku, Åbo Akademi University, and Biocenter Finland.

\section{COMPETING INTERESTS}

578 The authors declare no competing interests.

\section{FUNDING}

581 This study has been funded by The Academy of Finland (LS), Sigrid Juselius Foundation (LS), 582 Turku Doctoral Network in Molecular Biosciences (JJ, AJDS, JCL), Finnish Cultural Foundation 583 (JJ), Cancer Foundation Finland sr (JJ, LS), Åbo Akademi University Research Foundation (JJ, 584 MB), Tor, Joe and Pentti Borg Memory Foundation (JJ), Ida Montin's Foundation (JJ), Otto A. 585 Malm Foundation (JJ), the Medical Research Foundation Liv och Hälsa (JJ), K. Albin Johansson's

586 Foundation (JJ, AJDS), Center for International Mobility (AJDS), Agence Nationale Recherche 587 (Program SAMENTA ANR-13-SAMA-0008-01) (ADT, VM, DSD), Short Researcher Mobility 588 France Embassy/MESRI-Finnish Society of Science and Letters (VM), and CNRS/Project International de Coopération Scientifique PICS 2013-2015 (ADT, VM, DSD).

\section{DATA AVAILABILITY}

592 The original data are available at Gene Expression Omnibus (GEO) database under accession no. 593 GSE115973. 


\section{REFERENCES}

596 1. Gomez-Pastor R, Burchfiel ET, Thiele DJ (2018) Regulation of heat shock transcription factors and their roles in physiology and disease. Nat Rev Mol Cell Biol 19:4-19.

2. Vihervaara A, et al. (2013) Transcriptional response to stress in the dynamic chromatin environment of cycling and mitotic cells. Proc Natl Acad Sci USA 110:E3388-E3397. doi: 10.1073/pnas. 1305275110 .

3. Vihervaara A, et al. (2017) Transcriptional response to stress is pre-wired by promoter and enhancer architecture. Nat Commun 8:255. doi: 10.1038/s41467-017-00151-0.

5. Hartl FU, Bracher A, Hayer-Hartl M (2011) Molecular chaperones in protein folding and

4. Mahat DB, Salamanca HH, Duarte FM, Danko CG, Lis JT (2016) Mammalian heat shock response and mechanisms underlying its genome-wide transcriptional regulation. Mol Cell

8. Riva L, et al. (2012) Polyglutamine expanded huntingtin dramatically alters the genomewide binding of HSF1. J Huntingtons Dis 1:33-45.

615 9. Hahn J-S, Hu Z, Thiele DJ, Iyer VR (2004) Genome-wide analysis of the biology of stress responses through heat shock transcription factor. Mol Cell Biol 24:5249-5256. analysis reveals that active heat shock factor binding sites are mostly associated with nonheat shock genes in drosophila melanogaster. PLoS One 6:e15934. doi:10.1371/journal.pone.0015934.

11. Åkerfelt M, Morimoto RI, Sistonen L (2010) Heat shock factors: integrators of cell stress, development and lifespan. Nat Rev Mol Cell Biol 11:545-555.

12. Björk JK, et al. (2016) Heat-shock factor 2 is a suppressor of prostate cancer invasion. Oncogene 35:1770-1784.

13. Joutsen J, Sistonen L (2018) Tailoring of proteostasis networks with heat shock factors. Cold Spring Harb Perspect Biol. doi: 10.1101/cshperspect.a034066, Published online November 12, 2018.

14. Jaeger AM, Pemble CW 4th, Sistonen L, Thiele DJ (2016) Structures of HSF2 reveal 

23:147-154.

15. Sarge KD, Zimarino V, Holm K, Wu C, Morimoto RI (1991) Cloning and characterization of two mouse heat shock factors with distinct inducible and constitutive DNA-binding ability. Genes Dev 5:1902-1911.

16. Alastalo TP, et al. (1998) Stage-specific expression and cellular localization of the heat shock factor 2 isoforms in the rat seminiferous epithelium. Exp Cell Res 240:16-27.

18. Mezger V, Rallu M, Morimoto RI, Morange M, Renard JP (1994) Heat shock factor 2-like activity in mouse blastocysts. Dev Biology 166:819-822.

19. Elsing AN, et al. (2014) Expression of HSF2 decreases in mitosis to enable stressinducible transcription and cell survival. J Cell Biol 206:735-749.

22. Kallio M, et al. (2002) Brain abnormalities, defective meiotic chromosome synapsis and female subfertility in HSF2 null mice. EMBO J 21:2591-2601.

23. Chang Y, et al. (2006) Role of heat-shock factor 2 in cerebral cortex formation and as a regulatorof p35 expression. Genes Dev 20:836-847.

24. Ahlskog JK, et al. (2010) Anaphase-promoting complex/cyclosome participates in the acute response to protein-damaging stress. Mol Cell Biol 30:5608-56020.

26. Sandqvist A, et al. (2009) Heterotrimerization of heat-shock factors 1 and 2 provides a transcriptional switch in response to distinct stimuli. Mol Biol Cell 20:1340-1347.

27. Pirkkala L, Alastalo TP, Zuo X, Benjamin IJ, Sistonen L (2000) Disruption of heat shock factor 1 reveals an essential role in the ubiquitin proteolytic pathway. Mol Cell Biol 20:2670-2675.

28. Kawazoe Y, Nakai A, Tanabe M, Nagata K (1998) Proteasome inhibition leads to the activation of all members of the heat-shock-factor family. Eur J Biochem 255:356-362. 
663

664

665

666

667

668

669

670

671

672

673

674

675

676

677

678

679

680

681

682

683

684

685

686

687

688

689

690

691

692

693

694

695

696

29. Varshavsky A (2012) The ubiquitin system, an immense realm. Annu Rev Biochem 81:167-176.

30. Hershko A, Ciechanover A, Varshavsky A (1998) The ubiquitin system. Annu Rev Biochem 67:425-479.

31. Deshaies RJ (2014) Proteotoxic crisis, the ubiquitin-proteasome system, and cancer therapy. BMC Biology 12:94. doi: 10.1186/s12915-014-0094-0.

32. Chen D, Frezza M, Schmitt S, Kanwar J, Dou QP (2011) Bortezomib as the first proteasome inhibitor anticancer drug: current status and future perspectives. Curr Cancer Drug Targets 11:239-253.

33. Goldberg AL (2012) Development of proteasome inhibitors as research tools and cancer drugs. J Cell Biol 199:583-588.

34. Kisselev AF, Callard A, Goldberg AL (2006) Importance of the different proteolytic sites of the proteasome and the efficacy of inhibitors varies with the protein substrate. $J$ Biol Chem 281:8582-8590.

35. Rossi A, et al. (2014) The proteasome inhibitor bortezomib is a potent inducer of zinc fingerAN1-type domain 2a gene expression: Role of heat shock factor 1 (HSF1)- heat shock factor 2 (HSF2) heterocomplexes. J Biol Chem 289:12705-12715.36.

36. Shah SP, et al. (2016) Bortezomib-induced heat shock response protects multiple myeloma cells and is activated by heat shock factor 1 serine 326 phosphorylation. Oncotarget 5:59727-59741. doi: 10.18632/oncotarget.10847.

37. Lecomte S, et al. (2010) Roles of heat shock factor 1 and 2 in response to proteasome inhibition: consequence on p53 stability. Oncogene 29:4216-4224.

38. Sistonen L, Sarge KD, Morimoto RI (1994) Human heat shock factors 1 and 2 are differentially activated and can synergistically induce hsp70 gene transcription. Mol Cell Biol 14:2087-2099.

39. Sheldon LA, Kingston RE (1993) Hydrophobic coiled-coil domains regulate the subcellular localization of human heat shock factor 2. Genes Dev 7:1549-1558.

40. Ling Y, et al. (2002) PS-341, a novel proteasome inhibitor, induces Bcl-2 phosphorylation and cleavage in association with G2-M phase arrest and apoptosis. Mol Cancer Ther $1: 841-849$

41. Shinkawa T, et al. (2011) Heat shock factor 2 is required for maintaining proteostasis against febrile-range thermal stress and polyglutamine aggregation. Mol Biol Cell $22: 3571-3583$.

42. Guettouche T, Boellmann F, Lane WS, Voellmy R (2005) Analysis of phosphorylation of 
human heat shock factor 1 in cells experiencing a stress. BMC Biochem 6:4.

43. Kubbutat MH, Jones SN, Vousden KH (1997) Regulation of p53 stability by Mdm2. Nature 387:299-303.

44. Kallio PJ, Wilson WJ, O'Brien S, Makino Y, Poellinger L (1999) Regulation of the hypoxia-inducible transcription factor $1 \alpha$ by the ubiquitin-proteasome pathway. $J$ Biol Chem 274:6519-6525.

47. Sarge KD, Murphy SP, Morimoto RI (1993) Activation of heat shock gene transcription by

45. Kobayashi A, et al. (2004) Oxidative stress sensor Keap1 functions as an adaptor for Cul3based E3 ligase to regulate proteasomal degradation of Nrf2. Mol Cell Biol 24:7130-7139.

46. Sarge KD, Park-Sarge OK, Kirby JD, Mayo KE, Morimoto RI (1994) Expression of heat shock factor 2 in mouse testis: potential role as a regulator of heat-shock protein gene expression during spermatogenesis. Biol Reprod 50:1334-1343. heat shock factor 1 involves oligomerization, acquisition of DNA-binding activity, and nuclear localization and can occur in the absence of stress. Mol Cell Biol 13:1392-1407.

48. Ritchie ME, et al. (2015) Limma powers differential expression analyses for RNAsequencing and microarray studies. Nucleic Acids Res 43:e47. doi: 10.1093/nar/gkv007.

50. Dennis G, et al. (2003) DAVID: Database for Annotation, Visualization, and Integrated

51. Hirano S, Takeichi M (2012) Cadherins in brain morphogenesis and wiring. Physiol Rev

52. Peek SL, Mah KM, Weiner JA (2017) Regulation of neural circuit formation by protocadherins. Cell Mol Life Sci 74:4133-4157.

53. Stadler M, et al. (2018) Exclusion from spheroid formation identifies loss of essential cellcell adhesion molecules in colon cancer cells. Sci Rep 8:1151. doi: 10.1038/s41598-01819384-0.

54. Seong E, Yuan L, Arikkath J (2015) Cadherins and catenins in dendrite and synapse morphogenesis. Cell Adh Migr 9:202-213.

55. Fok JHL, et al. (2018) HSF1 is essential for myeloma cell survival and a promising therapeutic target. Clin Cancer Res 24:2395-2407.

56. Åkerfelt M, et al. (2008) Promoter ChIP-chip analysis in mouse testis reveals Y chromosome occupancy by HSF2. Proc Natl Acad Sci USA 105:11224-11229. 
enhancement of motility. J Cell Sci 128:1455-1464.

732 58. Korfanty J, et al. (2014) Crosstalk between HSF1 and HSF2 during the heat shock response in mouse testes. Int J Biochem Cell Biol 57:76-83.

59. Ozawa M, Kemler R (1998) Altered cell adhesion activity by pervanadate due to the dissociation of alpha-catenin from the E-cadherin catenin complex. J Biol Chem 273:6166-6170.

60. Kennedy BK, et al. (2014) Geroscience: linking aging to chronic disease. Cell 159:709713.

61. Walter P, Ron D (2011) The unfolded protein response: from stress pathway to homeostatic regulation. Science 334:1081-1086.

62. Ciryam P, et al. (2016) A transcriptional signature of Alzheimer's disease is associated with a metastable subproteome at risk for aggregation. Proc Natl Acad Sci USA 113:47534758.

744 63. Härmä V, et al. (2010) A comprehensive panel of three-dimensional models for studies of prostate cancer growth, invasion and drug responses. PLoS One 5:e10431. doi: 10.1371/journal.pone.0010431.

747 64. Rueden CT, et al. (2017) ImageJ2: ImageJ for the next generation of scientific image data. BMC Bioinformatics 18:529. doi: 10.1186/s12859-017-1934-z.

65. Bolte S, Cordelières FP (2006) A guided tour into subcellular colocalisation analysis in 
Figure 1. HSF2 is required for cell survival upon prolonged Bortezomib (BTZ) treatment. (A) Immunoblot analysis of HSF2 expression. U2OS WT cells were treated with indicated concentrations of BTZ for 6 or $22 \mathrm{~h}$. Control cells were treated with DMSO. Tubulin was used as a loading control. (B) Confocal microscopy images of HSF2 immunofluorescence staining. U2OS WT cells were plated on cover slips and treated with $25 \mathrm{nM} \mathrm{BTZ}$ for 6,10 , or $22 \mathrm{~h}$. Control cells were treated with DMSO. Samples were fixed and stained with anti-HSF2 antibody. DAPI was used for DNA detection. The overlay of HSF2 and DAPI maximum intensity projection signals is shown in merge. Scale bar $100 \mu \mathrm{m}$. (C) Immunoblot analysis of HSF2 expression in U2OS WT and HSF2 KO (2KO) cells. Tubulin was used as a loading control. (D) Bright-field microscopy images of WT and $2 \mathrm{KO}$ cells treated with indicated concentrations of BTZ for $22 \mathrm{~h}$. Control cells were treated with DMSO. Scale bar $100 \mu \mathrm{m}$. (E) Calcein AM assay of WT and 2KO cells treated as in D. Relative fluorescence was calculated against each respective control that was set to 1 . The data is presented as mean values of at least three independent experiments + SEM, ${ }^{*} \mathrm{p}<0.05$. (F) Immunoblot analysis of PARP-1. Cells were treated as in D. HSC70 was used as a loading control.

(G) Calcein AM assay of U2OS WT cells transfected with Scr or HSF2-targeting shRNA constructs [25] and treated with $25 \mathrm{nM} \mathrm{BTZ}$ for $22 \mathrm{~h}$. Relative fluorescence was calculated against each respective control that was set to 1 . The data is presented as mean values of three independent experiments + SEM, $* p<0.05$. (H) Immunoblot analysis of HSF2 and PARP-1. Cells were transfected and treated as in G. Tubulin was used as a loading control.

Figure 2. HSF2 is not required for the heat shock response during BTZ-induced proteotoxic stress. (A) A schematic overview of the RNA-seq experiment outline. U2OS WT and HSF2 KO (2KO) cells were treated with $25 \mathrm{nM} \mathrm{BTZ}$ for 6 or $10 \mathrm{~h}$. Control cells were treated with DMSO. After treatments, mRNA was extracted and analyzed by RNA-seq. Experiments were performed in biological quadruplets. The arrows depict comparison pairs. (B) Differentially expressed (DE) genes in each comparison pair were determined with Bioconductor R package Limma [48] (FC $\geq$ 3 , FDR $<0.001)$. The upregulated and downregulated genes in a given comparison pair are indicated with red and blue bars, respectively. (C) Normalized gene expression data for human

782 heat shock proteins, as defined in [49], was used to calculate the fold change of each gene in relation 783 WT control sample. The data is presented as a heatmap of $\log _{2}$ transformed values and it was generated with GraphPad Prism7. Examples of genes that exhibit a divergent expression pattern are framed. (D, E) mRNA expression levels of selected heat shock proteins (HSPA1A, HSP90AA1, 
HSPA6, and HSPB1) (D), HSP90 co-chaperones (PTGES3 and AHSA1), and stress-responsive ubiquitin genes $(U B B$ and $U B C)(E)$ determined with RNA-seq. The data is presented as mean values \pm SEM relative to WT control sample that was set to 1 .

Figure 3. HSF2 impacts the expression of genes associated with cadherin-mediated cell-cell adhesion. (A) A schematic overview of the U2OS WT and HSF2 KO (2KO) comparison pairs. (B) Differentially expressed (DE) genes in 2KO : WT comparison pairs (control, $6 \mathrm{~h}, 10 \mathrm{~h}$ ) were determined with Bioconductor R package Limma [48] (FC $\geq 3$, FDR $<0.001)$. The upregulated and downregulated genes are indicated with red and blue bars, respectively. (C) Gene ontology (GO) terms were analyzed with topGO and GOstats packages in Bioconductor R. Biological processes from each comparison pair were ranked according to their $\mathrm{p}$-values and the five most significantly changed GO terms are shown. The number of genes associated with a given term is indicated. (D) Venn diagrams presenting the interrelationship of significantly (FC $\geq 3$, FDR $<$ 0.001) upregulated or downregulated genes in $2 \mathrm{KO}:$ WT comparison pairs at control (orange), 6

$800 \mathrm{~h}$ (grey), and $10 \mathrm{~h}$ (green) time points. Diagrams were generated using the BioVenn web 801 application. (E) Gene term heatmap generated with DAVID Functional Annotation Clustering Tool 802 based on the 114 upregulated (left panel) and the 277 downregulated (right panel) genes in $2 \mathrm{KO}$ 803 cells in all treatment conditions as shown in panel D. Red and blue squares denote positive association between the gene and the keyword, Gene ontology (GO) term, or InterPro (IPR) term. Cluster enrichment score for upregulated gene cluster: 4.39; for downregulated gene cluster: 9.71.

Figure 4. HSF2 controls cellular adhesion properties through cadherin superfamily proteins.

(A) Normalized gene expression data from the RNA-seq analysis for cadherin superfamily genes, as defined in [51], was used to calculate the fold change of each gene in relation to respective expression in WT control sample. The data is presented as a heatmap of $\log _{2}$ transformed fold

811 changes and it was generated with GraphPad Prism7. N-cadherin and protocadherin gamma 812 subfamily A (PCDH $\gamma \mathrm{A}$ ) were chosen for further analyses. (B) Immunoblot analysis of HSF2, N813 cadherin, and the members of PCDH $\gamma$ A. U2OS WT and $2 \mathrm{KO}$ cells were treated with $25 \mathrm{nM}$ BTZ 814 for 6 or $10 \mathrm{~h}$. HSC70 was used as a loading control. (C) Cell aggregation assay of U2OS WT and $8152 \mathrm{KO}$ cells suspended in cell aggregation buffer supplemented with $3 \mathrm{mM} \mathrm{CaCl}_{2}\left(\mathrm{Ca}^{2+}\right)$ or $3 \mathrm{mM}$ 816 EDTA. Cells were rotated for $2.5 \mathrm{~h}$ at $37^{\circ} \mathrm{C}$ and visualized with bright-field microscopy. Scale bar $8171 \mathrm{~mm}$. The size of the aggregates was quantified with ImageJ. The data is presented as mean values 818 of three independent experiments + SEM. $* * * * p<0.0001$. (D) Confocal microscopy images of 819 U2OS WT and 2KO cells. Cells were cultured in 3D in Matrigel for 5, 8, and 13 days. At the 
indicated days, spheroids were fixed and F-actin was stained with Alexa 488-labeled phalloidin (green). DAPI was used to stain the nuclei (blue). Z-stacks of the spheroids were imaged with a spinning disc confocal microscope. The maximum intensity projection images represent the average spheroid size for each cell line at indicated time points from three biological repeats. Scale bar $10 \mu \mathrm{m}$. The volume of the spheroids was quantified with ImageJ with 3D Object Counter v2.0 plugin [65]. The data is presented as mean values of three independent experiments + SEM. ***p $<0.001$.

Figure 5. Impaired cell-cell contacts sensitize U2OS cells to BTZ-induced proteotoxic stress. $\mathrm{N}$-cadherin levels in U2OS $2 \mathrm{KO}$ cells were restored to those in WT cells by transiently transfecting 830 the cells with either Mock or N-cadherin plasmids co-expressing GFP (A-C), for details see 831 Materials and Methods. (A) Immunoblot analysis of HSF2 and N-cadherin. HSC70 was used as a 832 loading control. (B) Cell aggregation assay was performed as in Figure 4C. Cell aggregates were 833 imaged with bright-field (BF) and fluorescence filters (GFP). Scale bar $500 \mu \mathrm{m}$. (C) For 834 immunoblot analysis of HSF2 and PARP-1, cells were treated with $25 \mathrm{nM} \mathrm{BTZ}$ for $22 \mathrm{~h}$. Control cells were treated with DMSO. HSC70 was used as a loading control. The amount of cleaved PARP-1 relative to respective HSC70 level was quantified with ImageJ. The data is presented as mean values of three independent experiments $+\mathrm{SEM},{ }^{*} \mathrm{p}<0.05$. (D) A schematic overview of the calcium-depletion experiments. U2OS WT and $2 \mathrm{KO}$ cells were first treated with or without $25 \mathrm{nM}$ BTZ for $20 \mathrm{~h}$ in serum free growth medium after which the extracellular calcium was depleted with $3 \mathrm{mM}$ EDTA and BTZ treatment continued for $2 \mathrm{~h}$. Control cells were treated with DMSO. Cell viability was assessed with Calcein AM assay. Relative fluorescence was calculated against each respective control value that was set to 1 . The data is presented as mean values of three independent experiments $+\mathrm{SEM}, * * \mathrm{p}<0.01, * * * \mathrm{p}<0.001$. Statistical significance was determined with unpaired t-test. Cells were imaged with a bright-field microscope. Scale bar $100 \mu \mathrm{m}$.

Figure Supplement 1. HSF2 depletion sensitizes cells to proteotoxic stress independently of HSF1 phosphorylation on serine 326. (A) Immunoblot analysis of HSF2 and PARP-1. U2OS WT, 2KO, and HSF2 knock-out clone 2 (2KO\#2) cells were treated with $25 \mathrm{nM}$ BTZ for $22 \mathrm{~h}$.

849 Control cells were treated with DMSO. HSC70 was used as a loading control. (B) Bright-field 850 microscopy images of U2OS WT and HSF2 KO $(2 \mathrm{KO})$ cells treated with indicated concentrations of MG132 for $22 \mathrm{~h}$. Control cells were treated with DMSO. Scale bar $100 \mu \mathrm{m}$. (C) Calcein AM assay of WT and $2 \mathrm{KO}$ cells treated as in A. Relative fluorescence was calculated against each 
854

independent experiments $+\mathrm{SEM}, * \mathrm{p}<0.05$. (D) Immunoblot analysis of PARP-1. Cells were treated as in A. HSC70 was used as a loading control. (E) Immunoblot analysis of HSF2 and PARP1. U2OS WT and $2 \mathrm{KO}$ cells were starved in L-arginine free growth medium for $17 \mathrm{~h}$ after which the medium was supplemented with L-canavanine (Can) in indicated concentrations. Cells were treated in this growth medium for 3 or 6 h. HSC70 was used as a loading control. (F) Bright-field microscopy images of U2OS WT and 2KO cells treated as in E. Scale bar $100 \mu \mathrm{m}$ (G) Immunoblot analysis of HSF2, HSF1 and HSF1 pS326. Cells were treated with either $50 \mathrm{nM} \mathrm{BTZ}$ or $10 \mu \mathrm{M}$ MG132 (MG) for $6 \mathrm{~h}$. Tubulin was used as a loading control. Arrowheads denote hyperphosphorylation of HSF1 [47].

\section{Figure Supplement 2. RNA-sequencing was performed in WT and HSF2-depleted U2OS} cells. (A) Bright-field microscopy images of U2OS WT and HSF2 KO (2KO) cells treated with 25 nM BTZ for 6 or $10 \mathrm{~h}$. Control cells were treated with DMSO. Scale bar $100 \mu \mathrm{m}$. (B) Immunoblot analysis of HSF2, HSF1 and HSP70. GAPDH was used as a loading control. Arrowheads denote hyperphosphorylation of HSF1 [47]. (C) Spearman's correlation of RNA-seq sample replicates (n $=4)$.

Figure Supplement 3. HSF2-dependent genes are linked to cell adhesion. mRNA expression levels of COL16A1, COL18A1, LAMB1, and LAMA5 in control (C) and BTZ-treated WT and 2KO cells determined with RNA-seq. The data is presented as mean values $\pm \mathrm{SEM}$ relative to WT control sample that was set to 1 .

Figure Supplement 4. Cadherin misregulation and sensitization to proteotoxicity are not HSF2 knock-out cell clone specific. (A) Immunoblot analysis of HSF2 and N-cadherin in WT and 2KO\#2 U2OS cells. HSC70 was used as a loading control. (B) mRNA expression of desmocollin 2 (DSC2), protocadherin $\alpha 6$ (PCDHA6), and protocadherin $\alpha 10$ (PCDHA10) of cadherin superfamily in WT, $2 \mathrm{KO}$, and $2 \mathrm{KO} \# 2$ cells. The mRNA levels were quantified with qRTPCR. The data is presented as mean values of three independent experiments + SEM, ****p < 0.0001. (C) Cell aggregation assay of U2OS WT and 2KO\#2 cells suspended in cell aggregation buffer supplemented with $3 \mathrm{mM} \mathrm{CaCl}_{2}\left(\mathrm{Ca}^{2+}\right)$ or $3 \mathrm{mM}$ EDTA. Cells were rotated for $2.5 \mathrm{~h}$ at $37^{\circ} \mathrm{C}$ and visualized with bright-field microscopy. Scale bar $1 \mathrm{~mm}$. (D) Bright-field microscopy images of U2OS WT and 2KO\#2 cells cultured in 3D in Matrigel. The spheroids were imaged at days 5, 8, and 13. Scale bar $100 \mu \mathrm{m}$. 


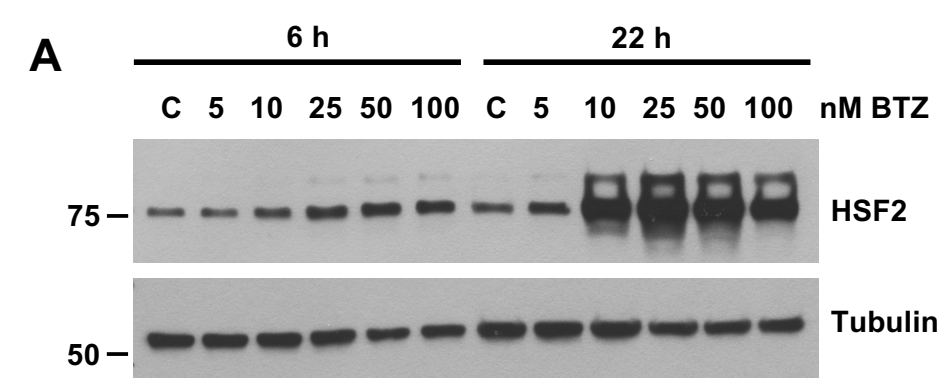

C
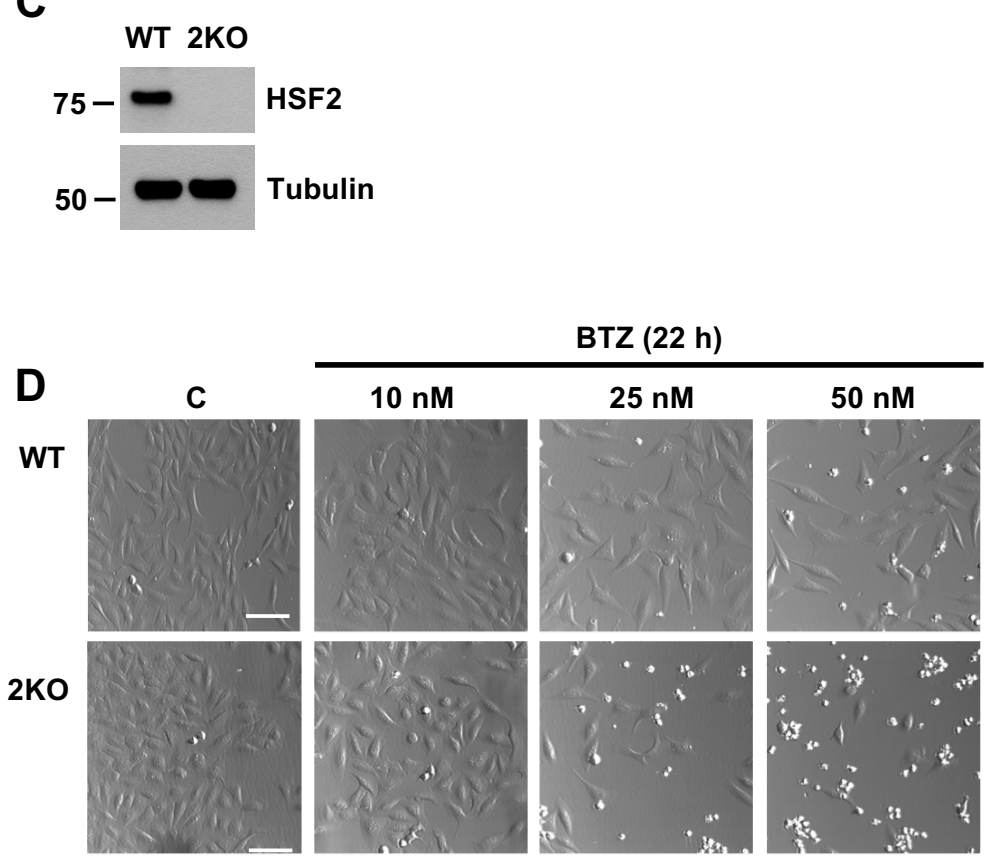

E

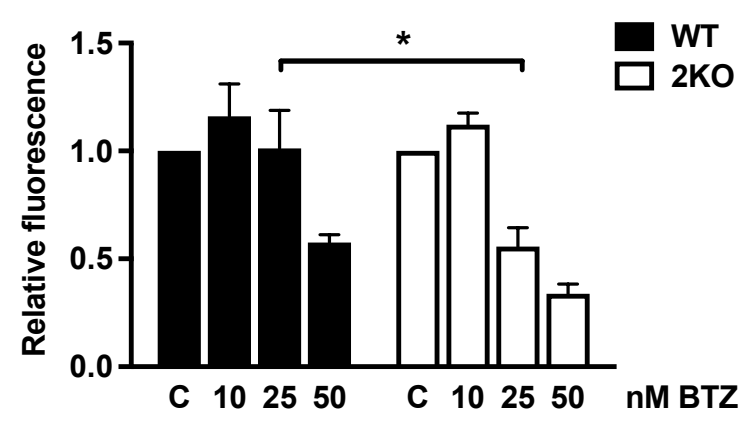

G

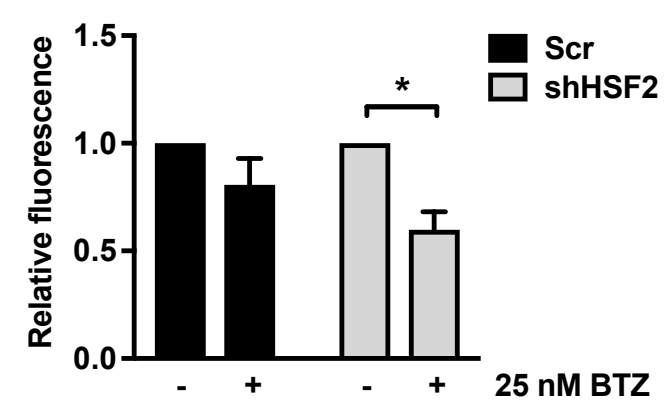

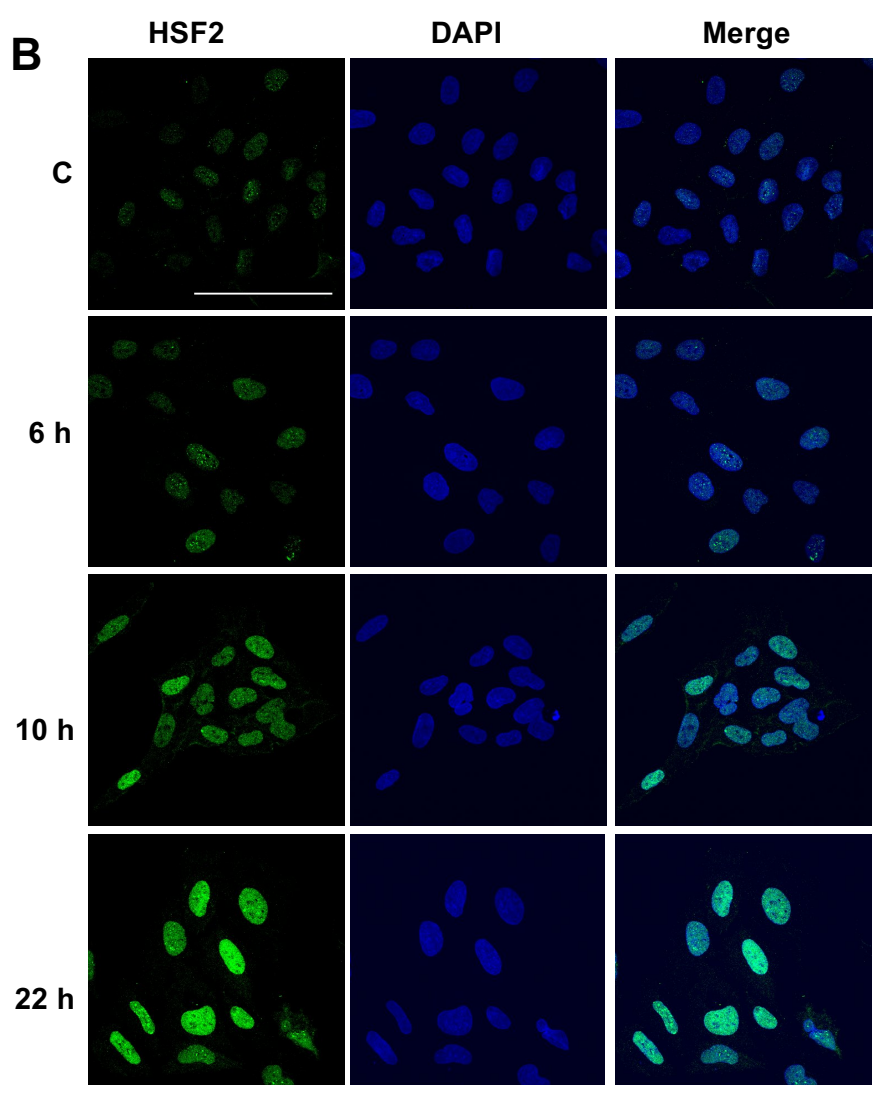

F
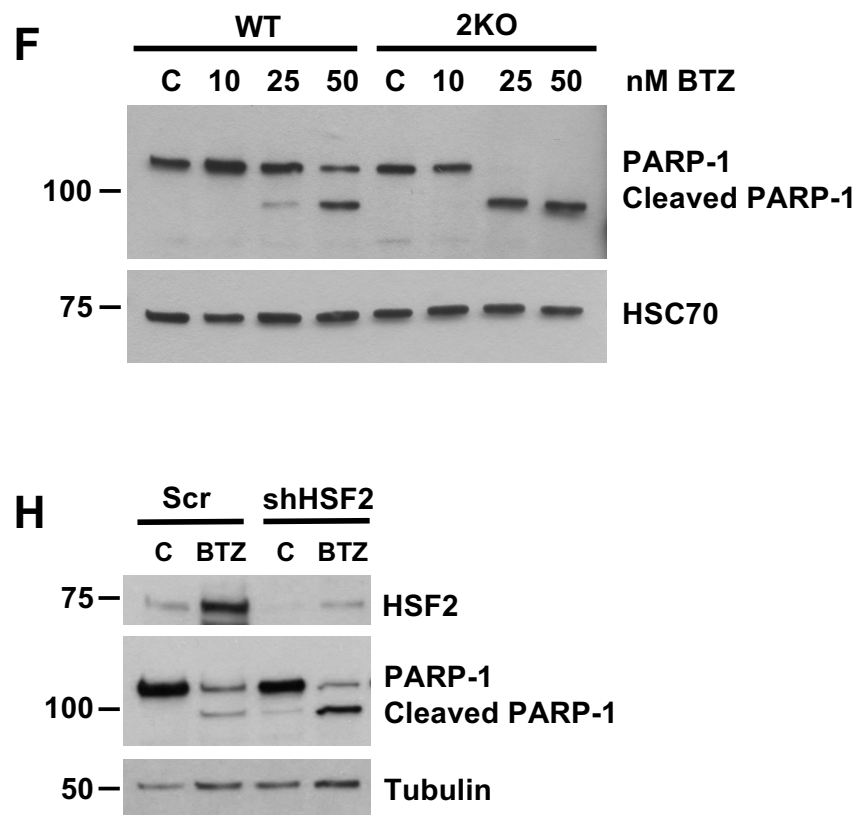


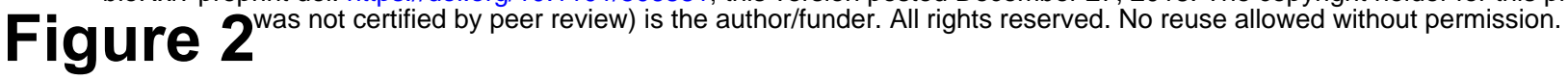

A

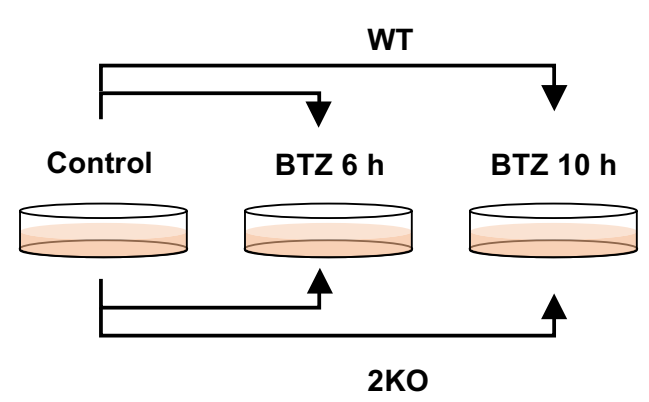

C

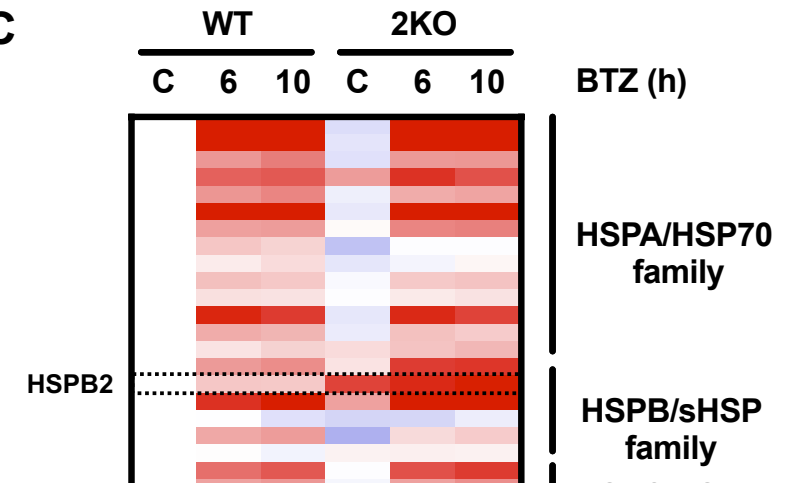

HSPC/HSP90

family

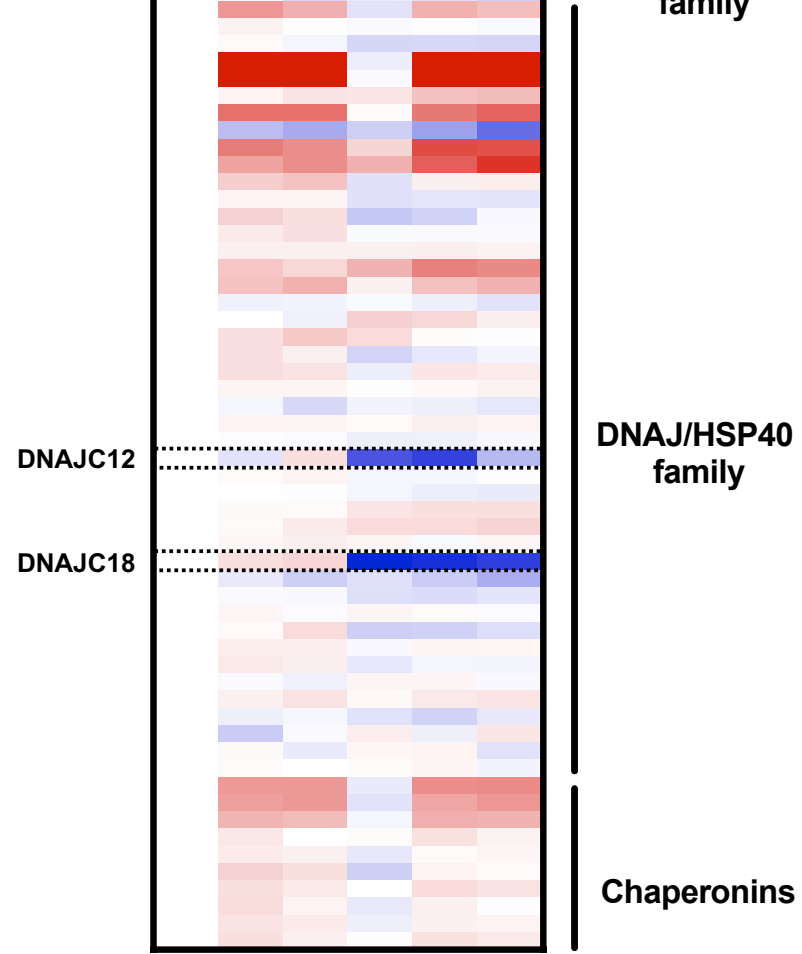

$\log _{2}$

Fold change
B
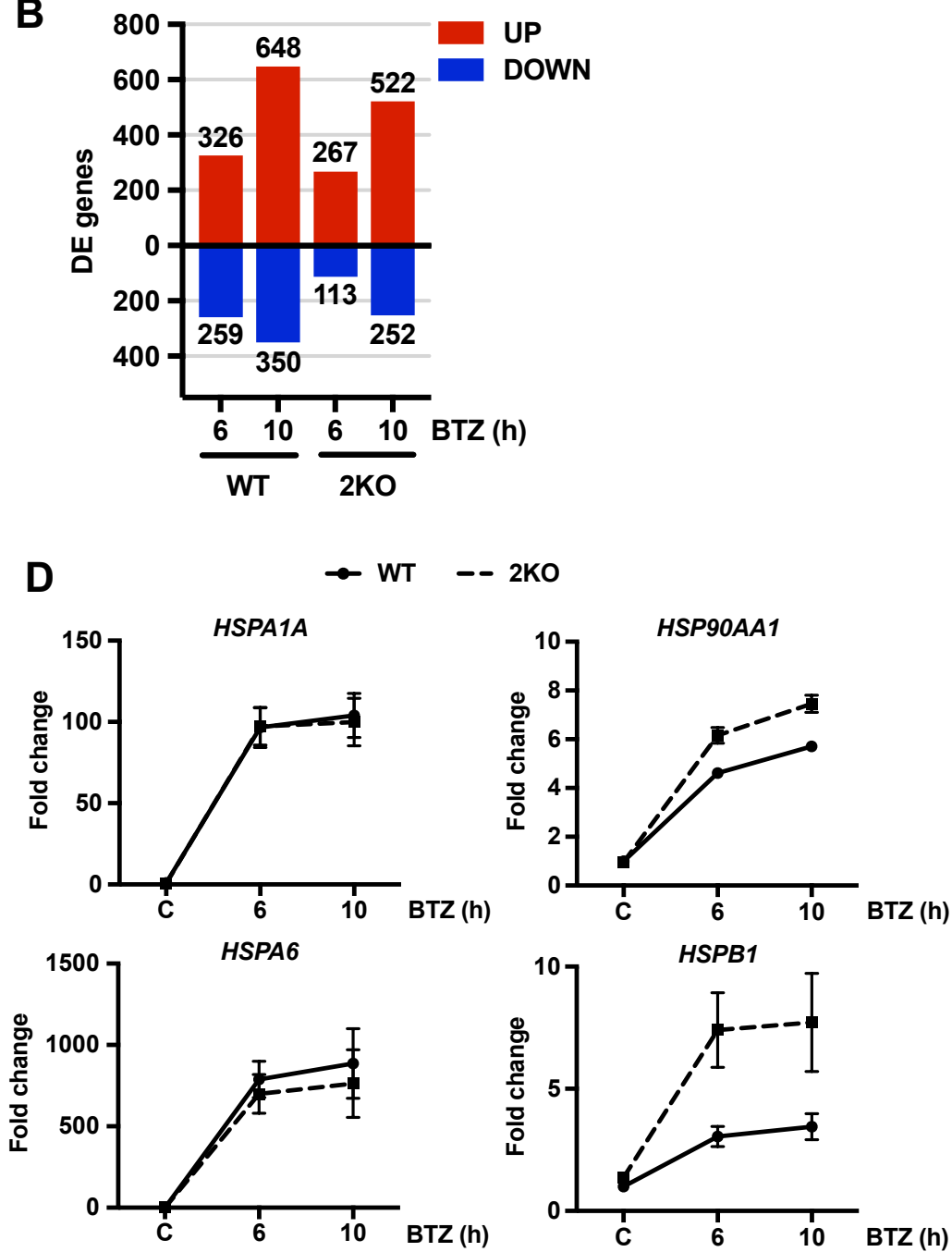

E
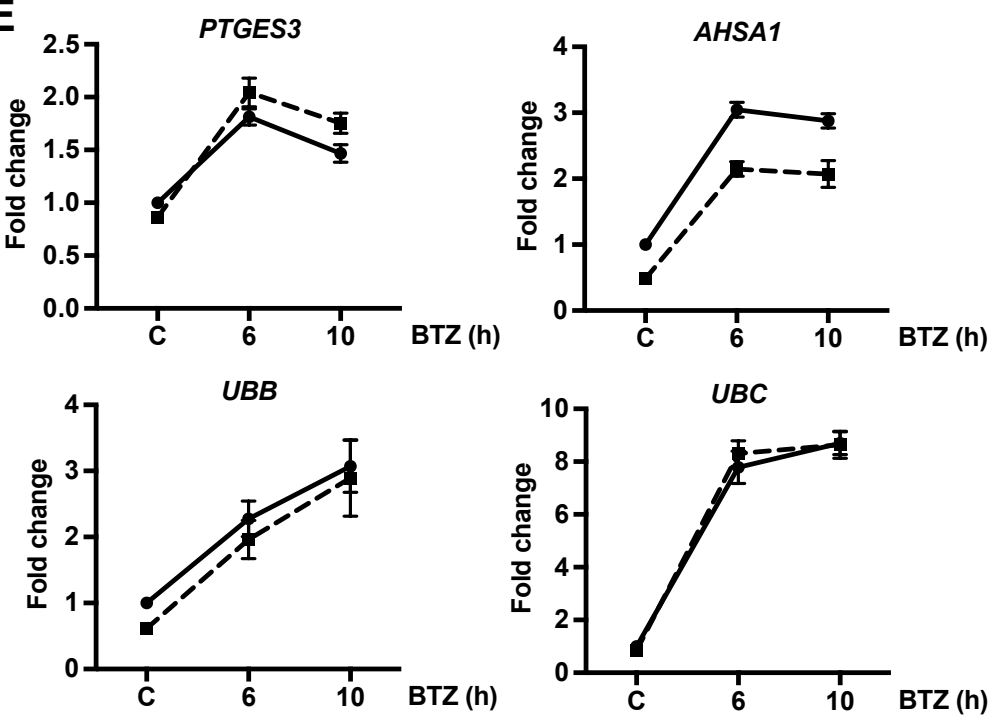
Figure 3

A

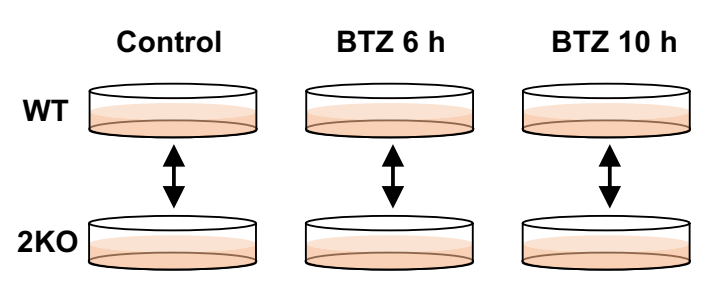

B

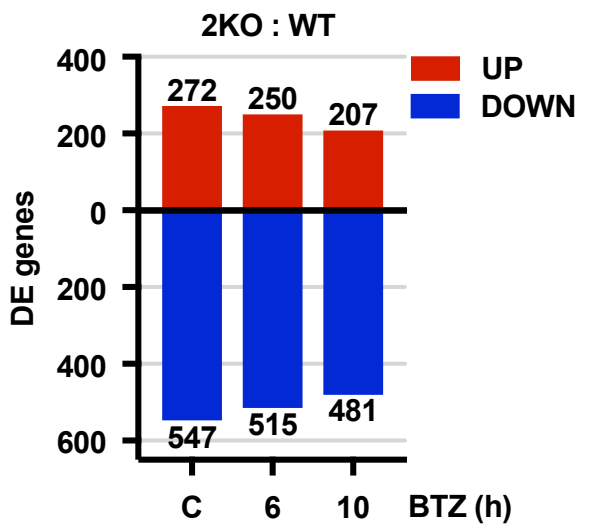

D

\section{C $2 \mathrm{KO}$ : C WT \\ $6 \mathrm{~h} 2 \mathrm{KO}: 6 \mathrm{~h}$ WT \\ 10 h 2 KO : 10 h WT}

UP
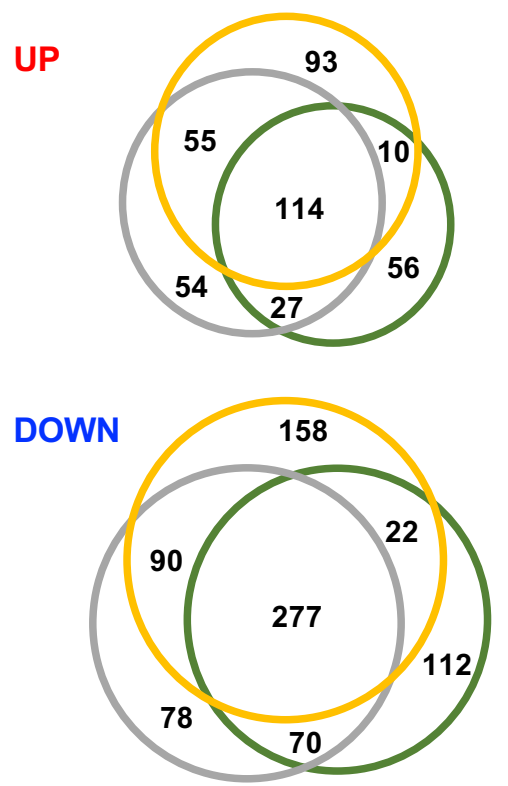

C

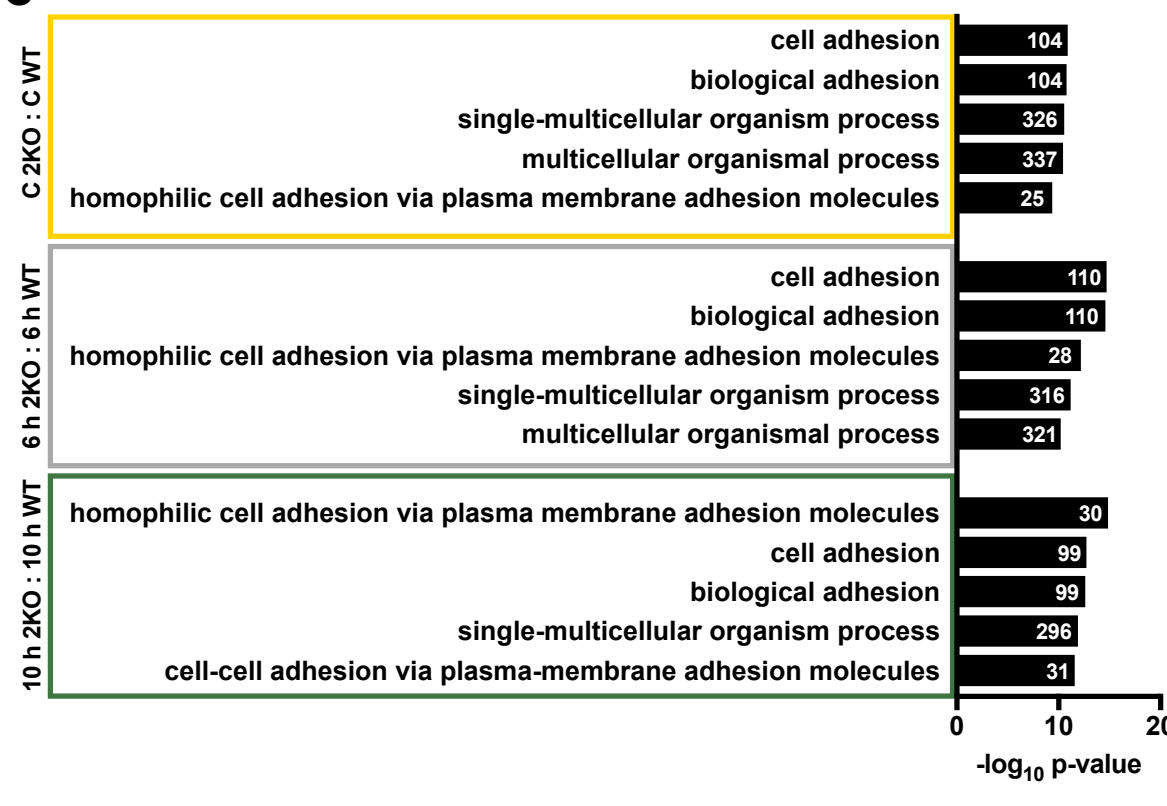

E $0^{0} \cdot B^{110}$ HAPLN4 COL18A1 MPZL2 MFAP4 FBLIM1 MPZL3 IGFBP7 LAMA5 OLR1 ITGB4 LAMB1 DCHS2 MSLN COL16A1 Enrichment score: 4.39

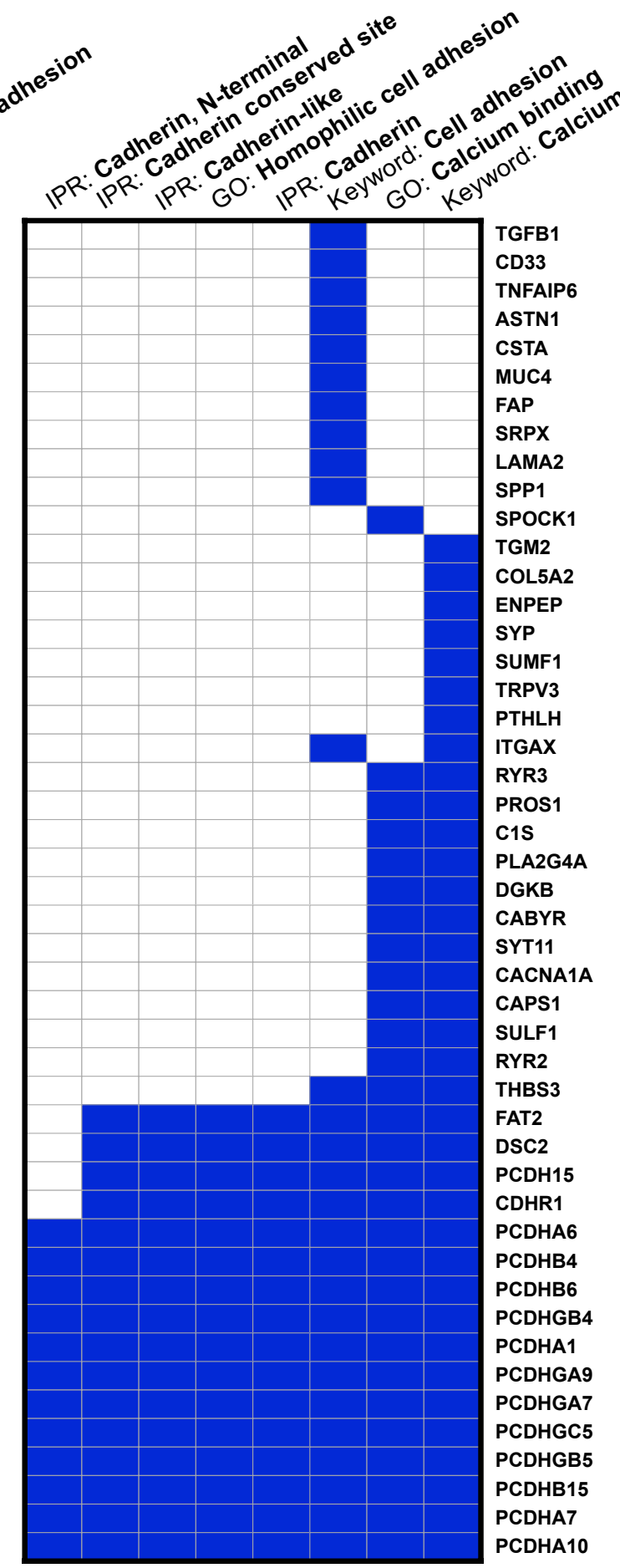


Figure 4

A

$$
\begin{array}{llllll}
\frac{\mathrm{WT}}{\mathrm{C}} & 6 & 10 & & \multicolumn{2}{c}{2 \mathrm{KO}} \\
\hline
\end{array}
$$

N-cadherin

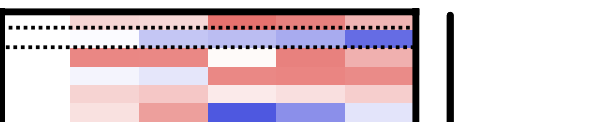

Classical cadherins

Desmosomal cadherins

Clustered protocadherins

(PCDH $\alpha, \mathbf{P C D H} \beta, \mathbf{P C D H} \gamma)$

Non-clustered protocadherins

CDH23-PCDH15

Fat-Dachsous

| Flamingo-Celsrs

I Calsyntenins
B

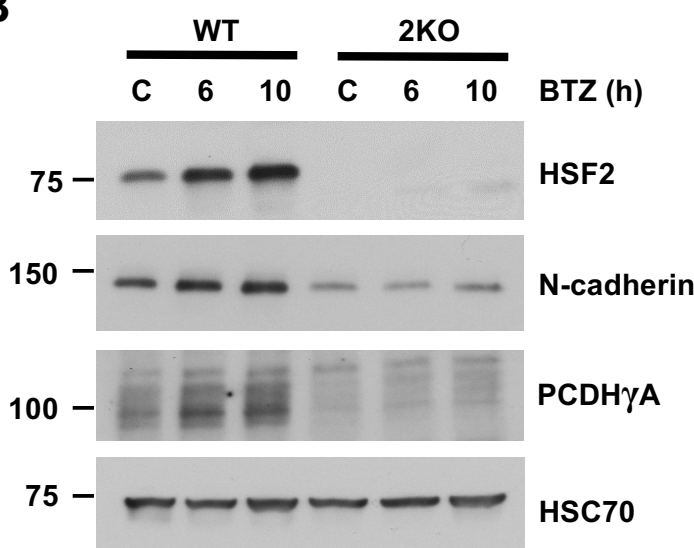

C
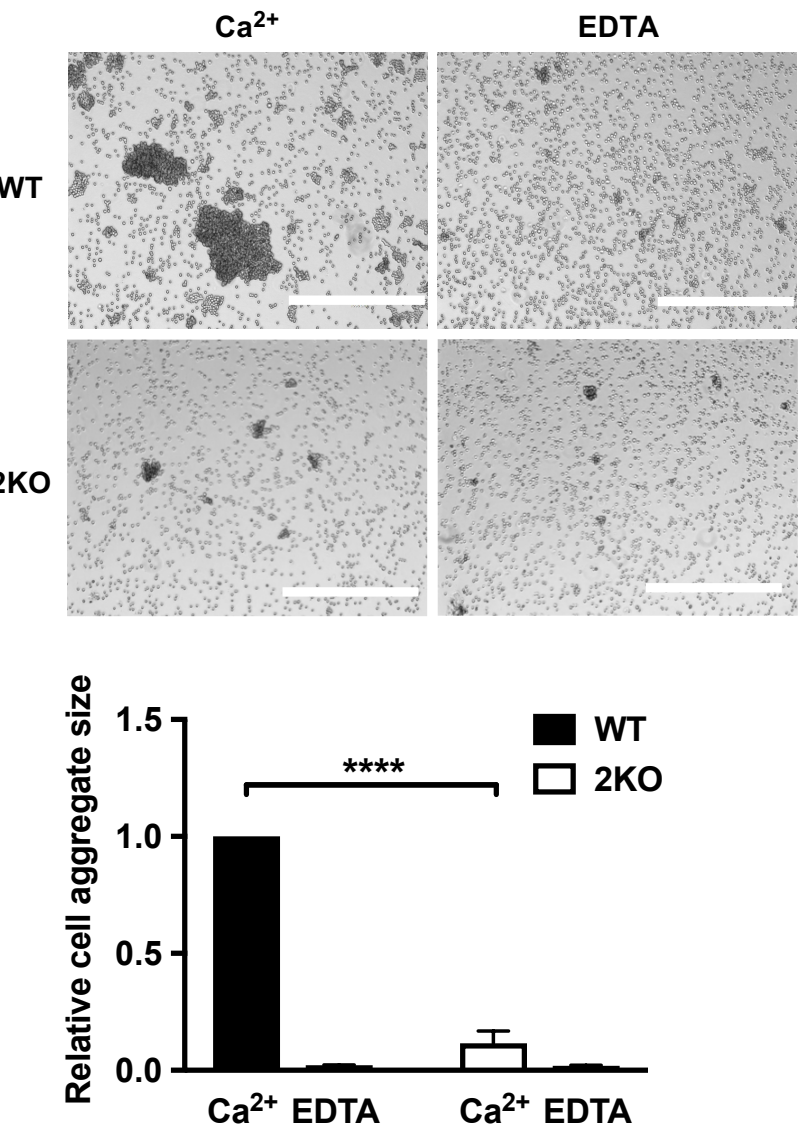

D

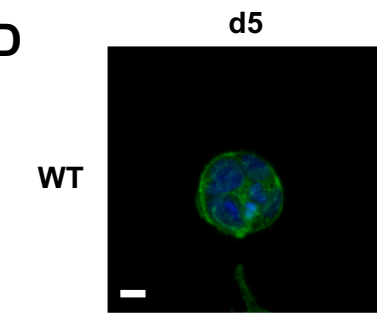

$2 \mathrm{KO}$ d8
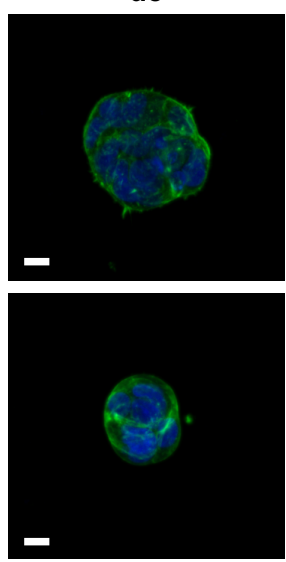

d13
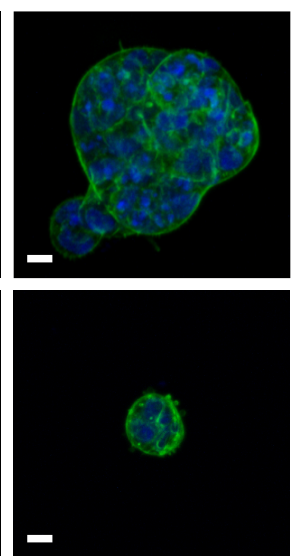

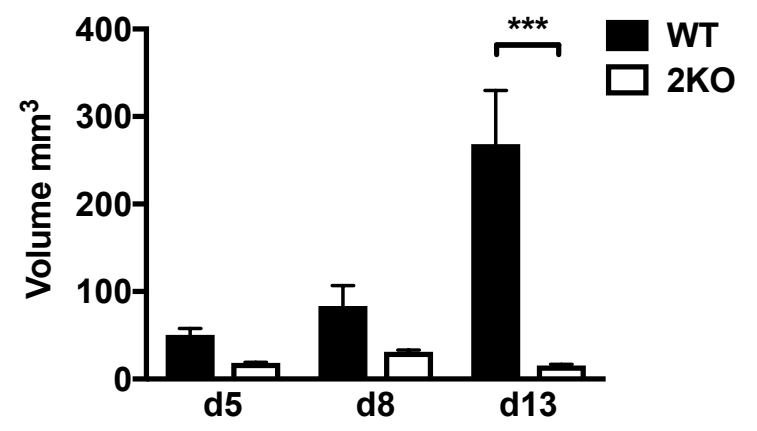




\section{Figure 5}

A

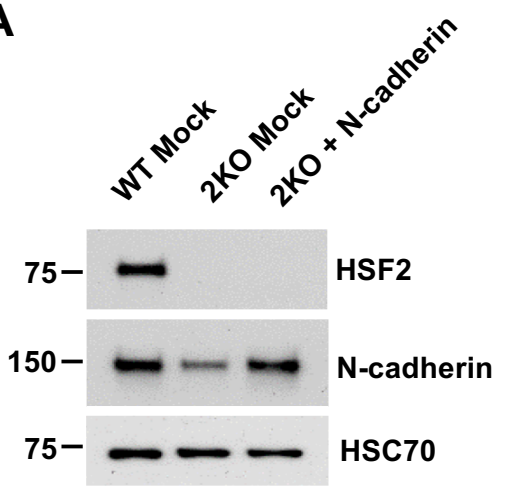

B
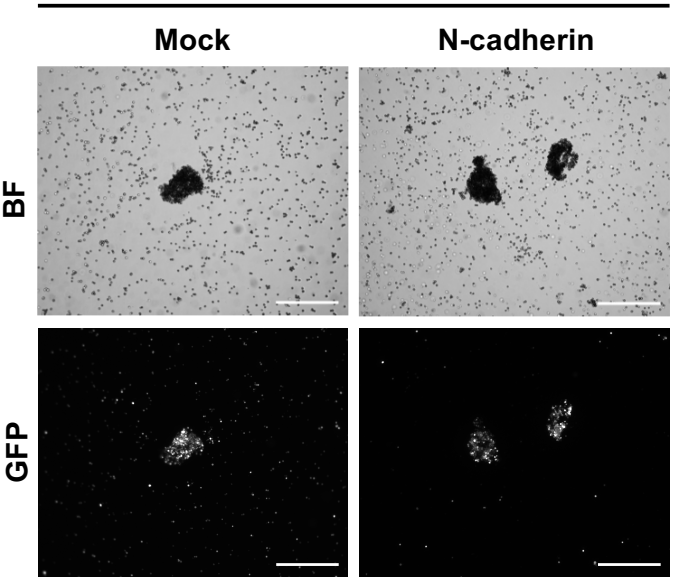

WT

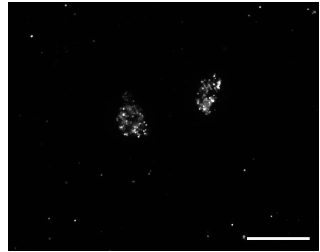

$2 \mathrm{KO}$

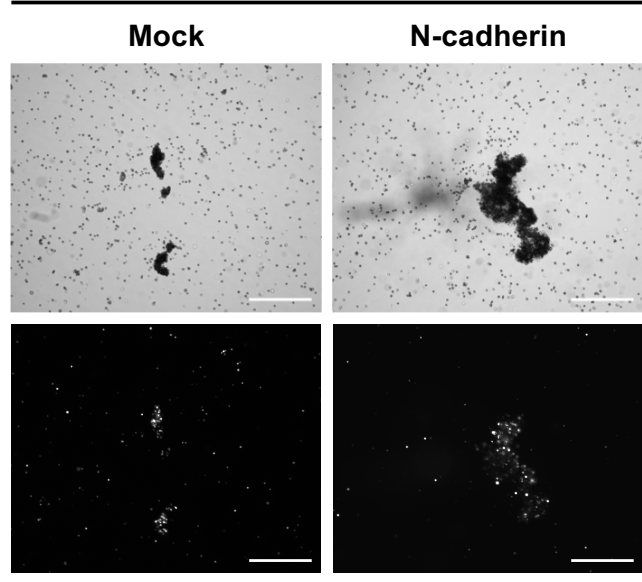

C
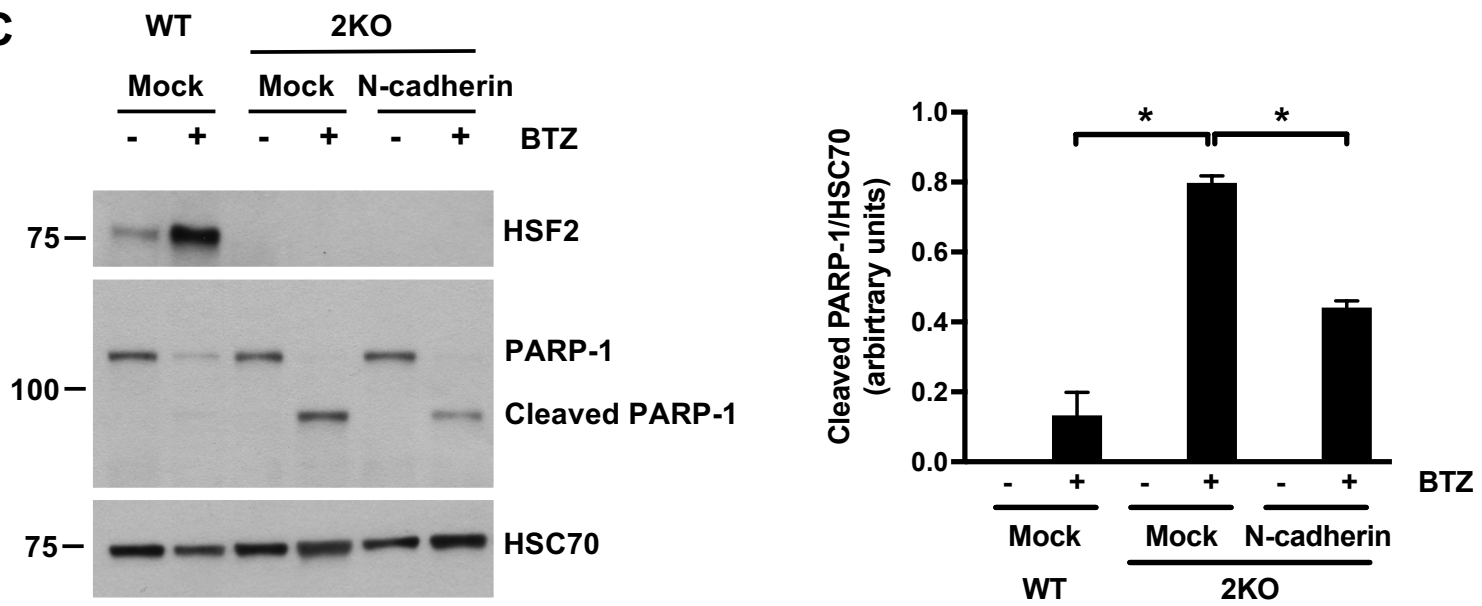

D

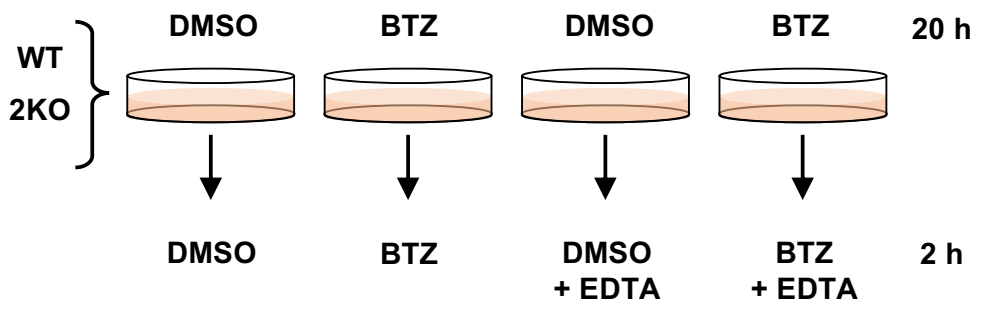

DMSO

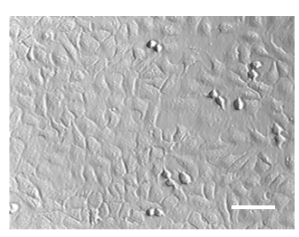

2KO

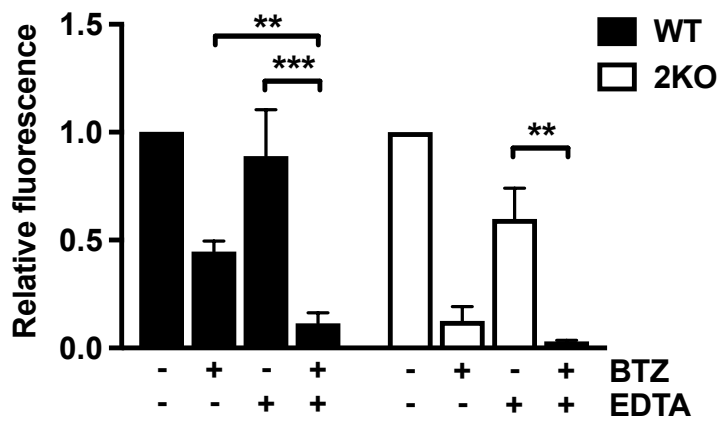

BTZ

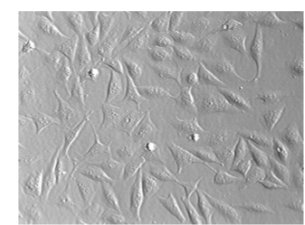

DMSO

+ EDTA

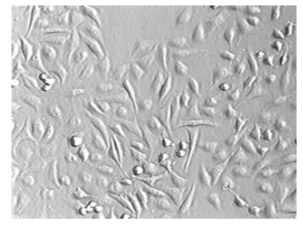

BTZ

+ EDTA
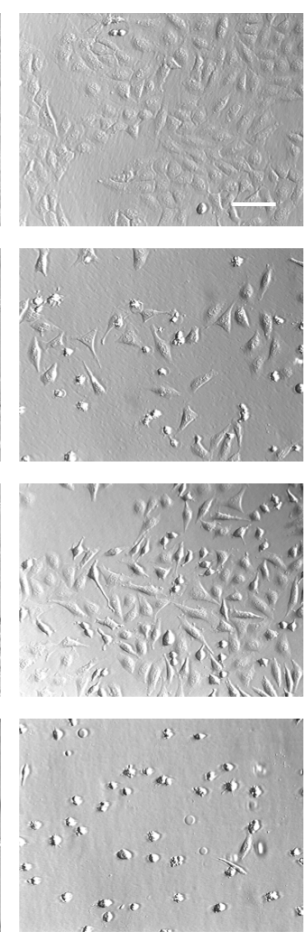
Figure Supplement 1

A

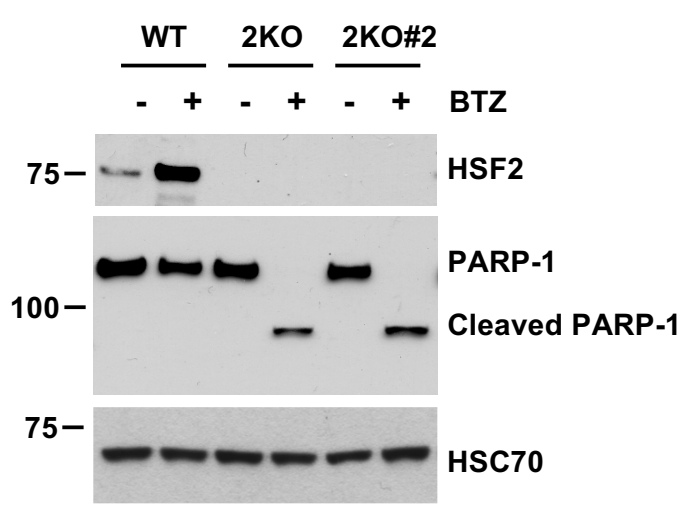

B

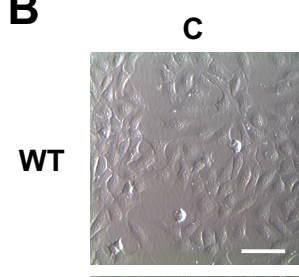

2KO

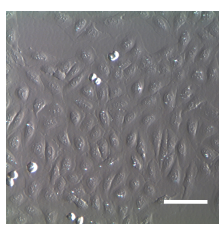

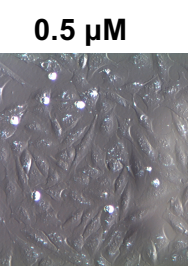
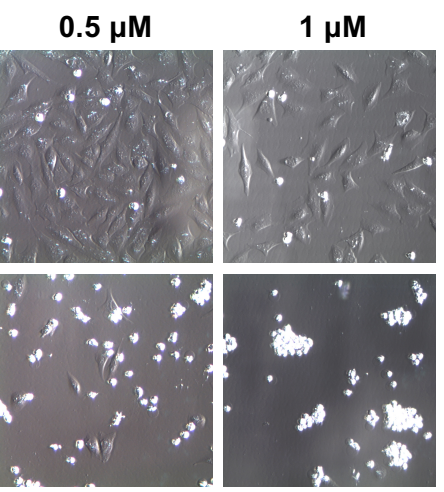

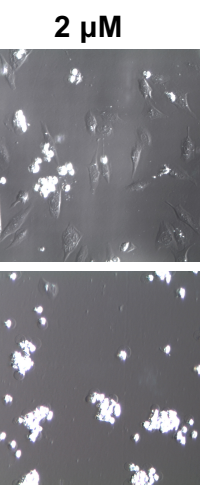

MG132

D
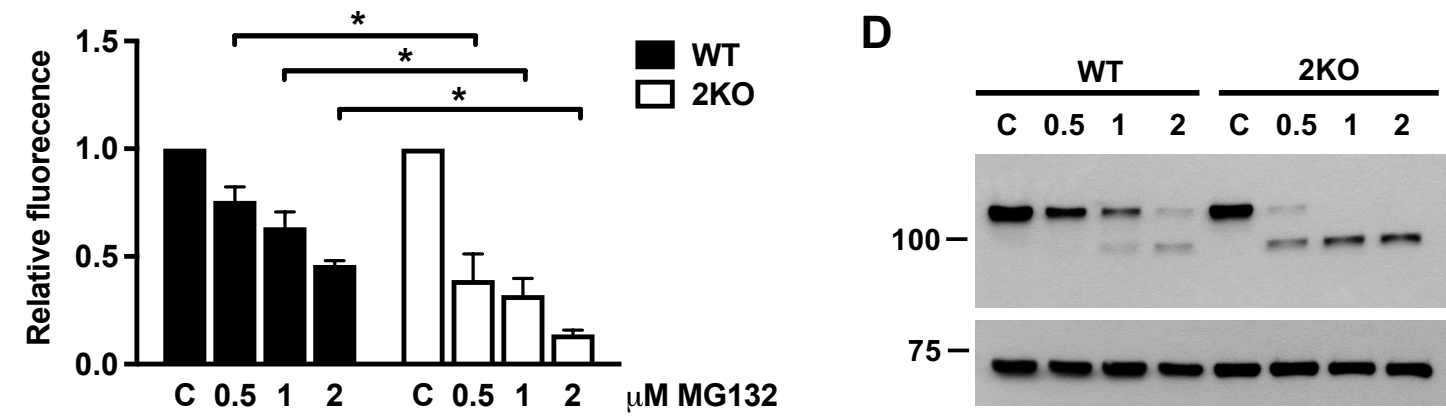

HM MG132

PARP-1

Cleaved PARP-1

HSC70

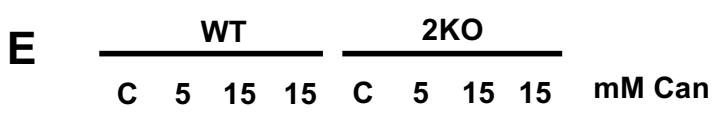

$\mathbf{F}$

$5 \mathrm{mM}$ Can

$15 \mathrm{mM}$ Can

$3 \mathrm{~h}$

$15 \mathrm{mM}$ Can
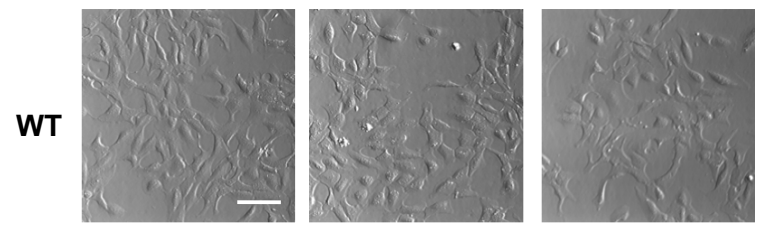

$6 \mathrm{~h}$

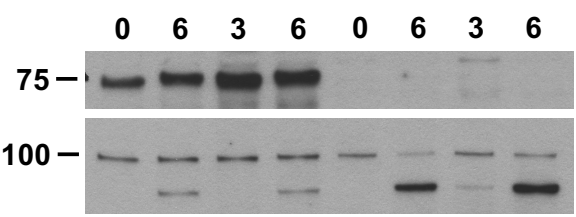

HSF2

PARP-1

Cleaved PARP-1

HSC70

2KO

G $\quad \frac{W T}{C \quad B T Z} \frac{2 K O}{C \quad B T Z}$
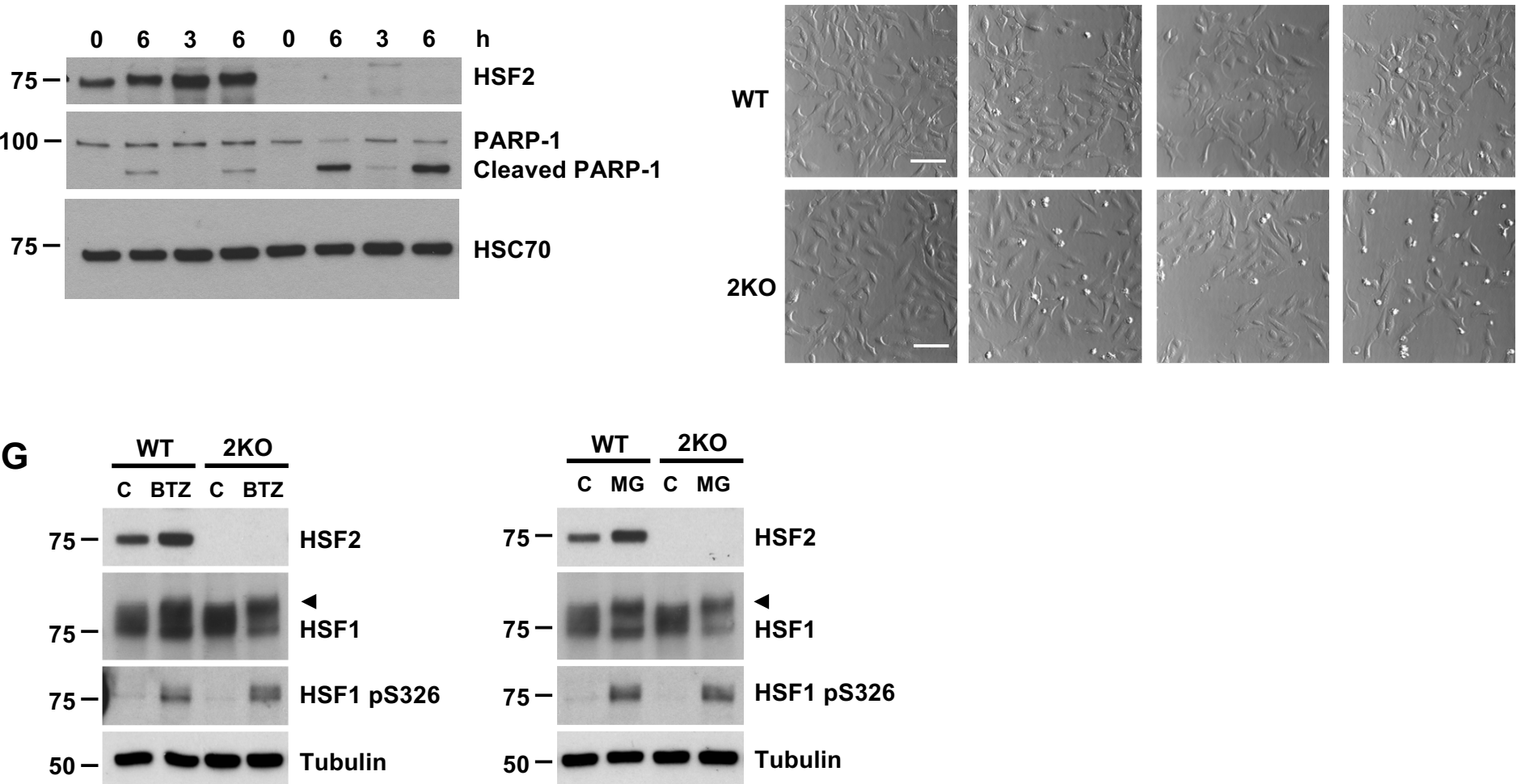


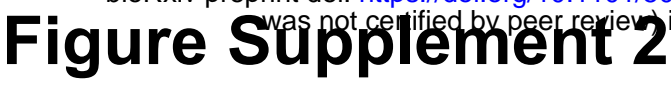

A

C

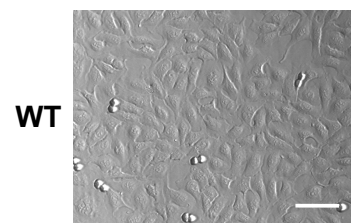

2KO

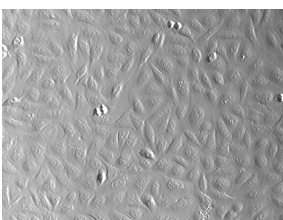

BTZ 6 h
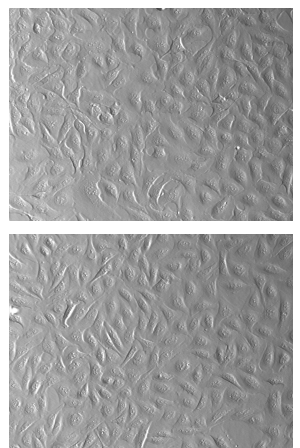

BTZ $10 \mathrm{~h}$

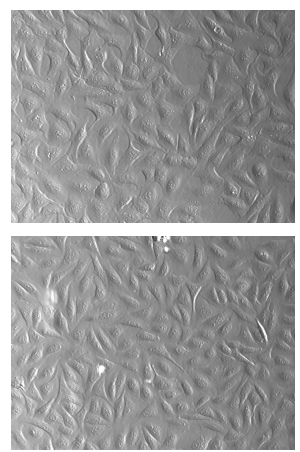

B

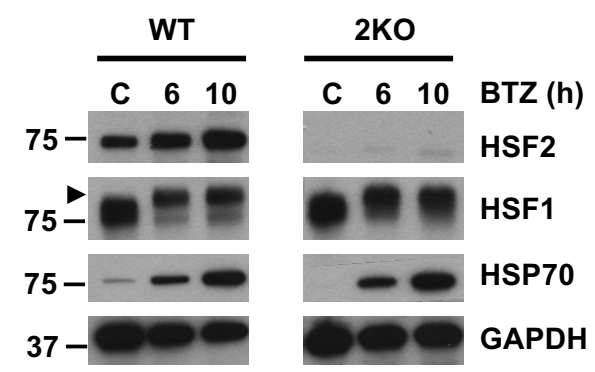

C

\begin{tabular}{|c|c|c|c|c|c|c|}
\hline Group & Name & minCor & meanCor & medianCor & maxcCor & corSD \\
\hline \hline 1 & WT C & 0,972 & 0,975 & 0,975 & 0,976 & 0,002 \\
\hline 2 & WT 6 h & 0,971 & 0,972 & 0,972 & 0,973 & 0,001 \\
\hline 3 & WT 10 h & 0,972 & 0,972 & 0,972 & 0,973 & 0,001 \\
\hline 4 & 2KO C & 0,973 & 0,974 & 0,973 & 0,975 & 0,001 \\
\hline 5 & 2KO 6 h & 0,972 & 0,973 & 0,973 & 0,974 & 0,001 \\
\hline 6 & 2KO 10 h & 0,972 & 0,973 & 0,973 & 0,974 & 0,001 \\
\hline
\end{tabular}




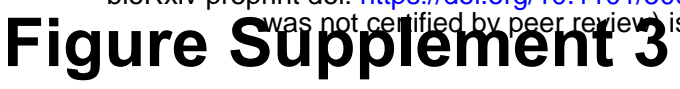
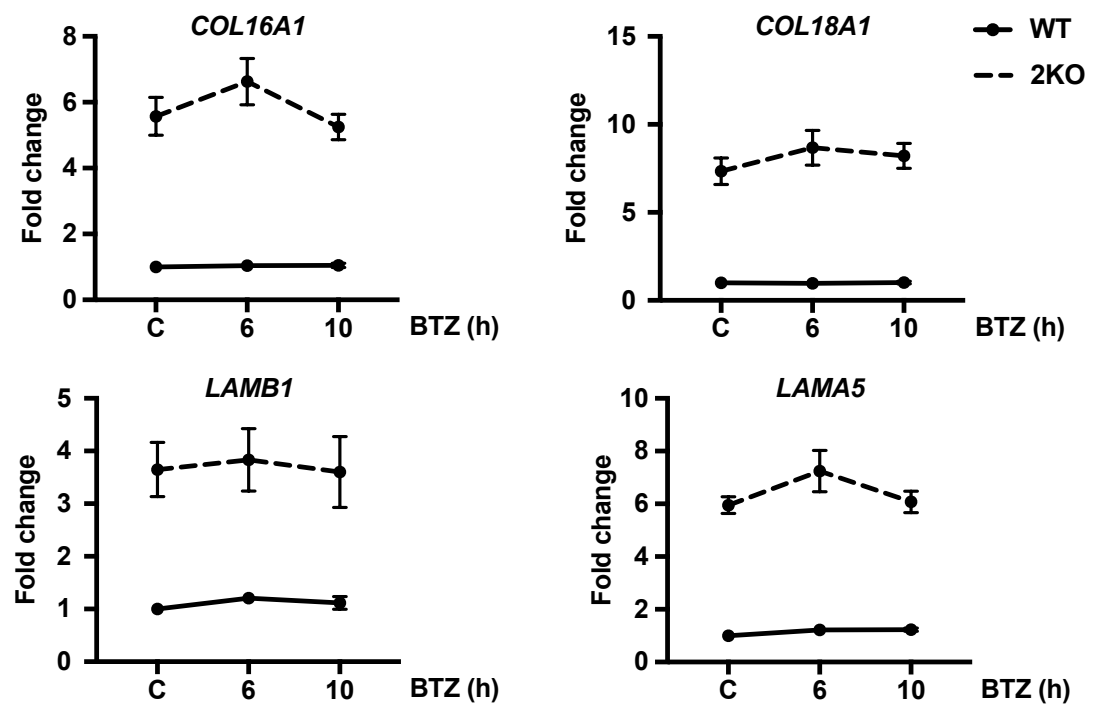

was not certified by peer review) is the author/funder. All rights reserved. No reuse allowed without permission.

\section{Figure Supplement 4}

A

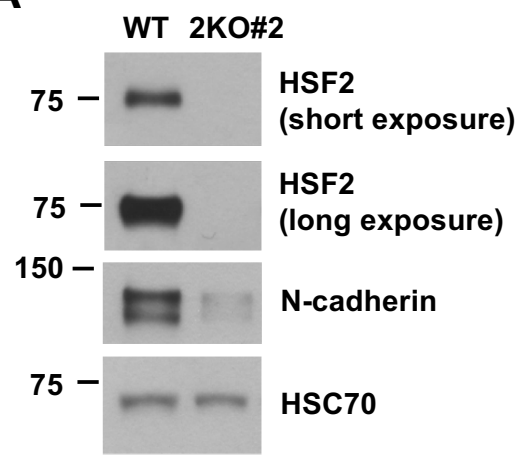

C

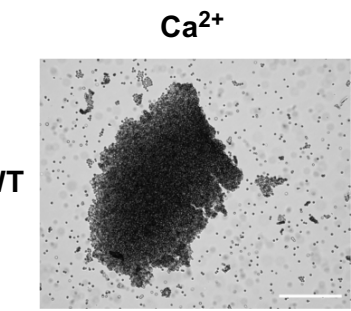

2KO\#2

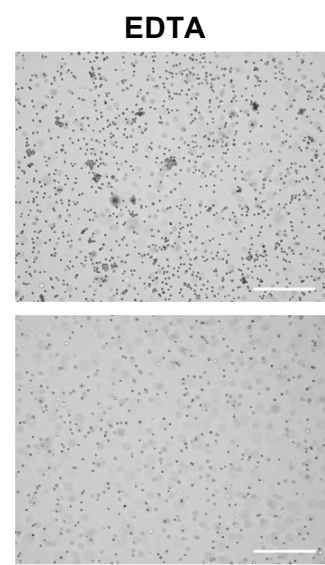

B

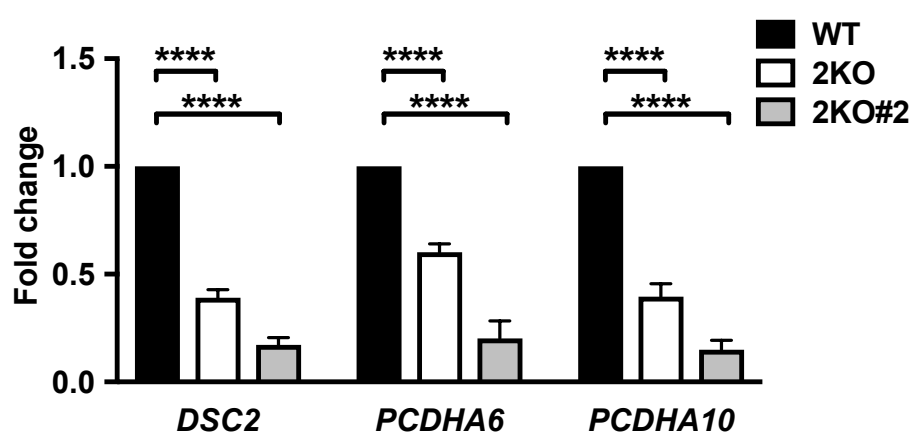

D

d5

d8
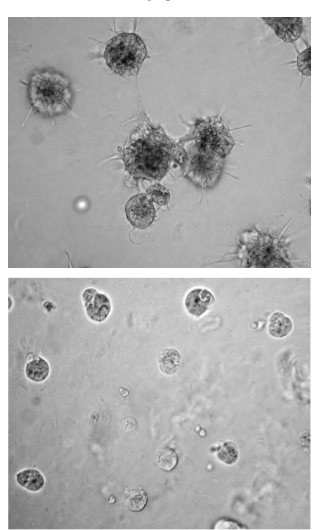

d13

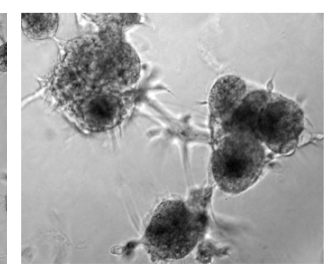

2KO\#2
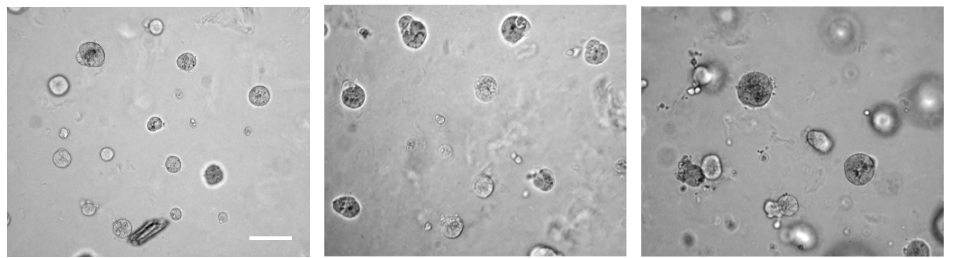